\title{
Fragile Networks: \\ Identifying Vulnerabilities and Synergies in an Uncertain Age
}

\author{
Anna Nagurney \\ Department of Finance and Operations Management \\ Isenberg School of Management \\ University of Massachusetts \\ Amherst, Massachusetts 01003 \\ Qiang Qiang \\ Management Division \\ Pennsylvania State University \\ Great Valley School of Graduate Professional Studies \\ Malvern, Pennsylvania 19355 \\ April 2010; revised May 2010
}

International Transactions in Operational Research 19: (2012), pp 123-160.

\begin{abstract}
This paper provides an overview of some of the recent developments in the assessment of network vulnerability and robustness through appropriate tools that assist in the quantification of network efficiency / performance and the identification of the importance of network components, such as nodes and links. We demonstrated how rigorously constructed and well-defined network measures can capture not only the network topology underlying a particular critical system, but also the underlying behavior of decision-makers, the resulting flows, and induced costs in the reality of demands for resources, whether fixed or elastic (price-dependent). In addition, we reviewed how to determine the synergy associated with network integration, with a focus on supply chains, as may occur not only in corporate applications, such as in mergers and acquisitions, but also in humanitarian ones, as in the case of the creation of teams and partnerships for humanitarian logistics.

We illustrated the concepts and tools in this paper, which are based on numerous publications, through a spectrum of applications and numerical examples. Since the number of disasters is growing globally, it is imperative to have transparent, well-understood, and appropriate tools for the determination of network vulnerability and robustness, since critical infrastructure networks from transportation, telecommunications, supply chains, to financial and electric power ones, provide the ties that bind our economies and societies together. Local disruptions can have global impacts. Only when network components are identified as to their importance and rankings can decision-makers and policy analysts as well as planners and engineers understand in an objective way which components should be maintained and
\end{abstract}


protected the most with implications for disaster and emergency preparedness as well as national security.

Key words: networks, efficiency measure, performance assessment, network robustness, network vulnerability, network fragility, transportation, supply chains, Internet, financial networks, electric power, smart grid, critical infrastructure, emergency and disaster preparedness, mergers and acquisitions, humanitarian logistics, synergy, variational inequalities, user-optimization, system-optimization 


\section{Introduction}

Networks provide the infrastructure upon which the functioning of our economies and societies depend. Networks that form the physical backbones of the modern age include: transportation networks that support the flows of vehicles from origins to destinations; manufacturing and logistical networks that enable the transformation of raw materials and supplies into finished products; energy networks that produce and transmit essential fuels, and the Internet that has transformed the ways in which we communicate, work, and conduct a myriad of business and social activities. Coupled with such physical networks are complex networks such as supply chains, financial networks, social networks, knowledge networks, and a plethora of economic networks, as well as networks, under development, such as the smart grid.

Modern networks vary in size, scale, and scope, from large-scale congested urban transportation networks on which drivers interact to airline networks that circle the globe to new railways that are being built in Asia to haul passengers and/or freight to relatively new social network media such as Facebook and Twitter that have emerged as communication forums in times of peace as well as disasters. The novelty of networks is that they are pervasive, providing the medium for connectivity of our societies and economies. Hence, the scientific understanding of networks has emerged as a notable area of both scholarly research and practice.

Methodologies, such as optimization techniques, game theory, variational inequalities, and dynamical systems, to name just a few, have advanced network theory, models, and algorithms, and have developed into a powerful and dynamic mechanism for abstracting complex decision-making behavior on networks, with the associated nodes, links, and induced flows. Rigorous network-based tools enable the analysis of complex problems, their solution, and provide managerial insights. Indeed, today, the formal study of networks spans many disciplines, with operations research, since its inception as a discipline, being, unarguably, a major contributor to the science of networks, over the past 70 years (see, e.g., König (1936), Kantorovich (1939), Hitchcock (1941), Koopmans (1947), Dantzig (1951), Beckmann, McGuire, and Winsten (1956), Ford and Fulkerson (1962), Sheffi (1985), Ahuja, Magnanti, and Orlin (1993), Ran and Boyce (1996), Nagurney and Siokos (1997), Nagurney (1999, 2000, 2006a), Nagurney and Dong (2002), Roughgarden (2005), Newman, Barabási, and Watts (2006), Daniele (2006), and Nagurney and Qiang (2009a)).

The subject of networks has garnered renewed interest and has attracted researchers and practitioners from physics, computer science, engineering, sociology, and even biology, due, 
in part, to a spectrum of catastrophic events such as 9/11, the North American electric power blackout in 2003, Hurricane Katrina in 2005, the Minneapolis bridge collapse in 2007, the Mediterranean cable disruption in 2008, Cyclone Nargis and the Sichuan Earthquake in China in 2008, the H1N1 pandemic in 2009, the earthquakes in Haiti and Chile in 2010, among others, all of which have drawn great attention to network vulnerability and fragility (cf. Nagurney, Yu, and Qiang (2009)).

The number of disasters is increasing globally, as well as the number of people affected by disasters, posing new challenges for emergency and disaster preparedness and for the critical network infrastructure itself from transportation to telecommunications. For example, between 2000 and 2004 the average annual number of disasters was $55 \%$ higher than in the period 1994 through 1999, with 33\% more humans affected in the former period than in the latter (cf. Balcik and Beamon (2008) and Nagurney and Qiang (2009a)). The International Strategy for Disaster Reduction (2006) found that approximately 150 million people required assistance, because of disasters, in 2005, with 157 million requiring assistance in 2006. Furthermore, according to Braine (2006), from January to October 2005 alone, an estimated 97,490 people were killed in disasters globally; 88,117 of them lost their lives because of natural disasters. As a specific illustration, the earthquake in Haiti that struck on January 12, 2010, affected one third of the country's population and resulted in the deaths of tens of thousands of its citizens with numerous others sustaining severe injuries. The earthquake profoundly demonstrated the vulnerability of the country's transportation, communication, and building infrastructures. Although definitions of what constitutes a disaster may differ (see, e.g., Federal Emergency Management Agency (1992) and the Emergency Events Database (2008)), the common thread is that a disaster has a catastrophic impact on a region's or even a nation's resources and on human lives.

The recent theories of scale-free and small-world networks in complex network research have enhanced our understanding of some of the behavior and the vulnerability of particular real-world networks (see Amaral et al. (2000), Chassin and Posse (2005), and Holmgren (2007)). Nevertheless, the majority of network vulnerability studies have focused on the topological characteristics of networks, such as the connectivity or the shortest path length of the network (see, e.g., Callaway et al. (2000) and the references therein). Although the topological structure of a network provides information regarding network vulnerability, the flow on a network is also an important indicator, as are the operational and economic aspects, such as the flow-induced costs, and the behavior of users both prior and post any disruptions. According to Barabási (2003), "To achieve that [understanding of complexity] we must move beyond structure and topology and start focusing on the dynamics that take 
place along the links. Networks are only skeletons of complexity, the highways for various processes that make our world hum."

For example, Latora and Marchiori (2001, 2002, 2004) proposed a network efficiency measure that exhibited advantages over several existing network measures and applied their measure to study the (MBTA) Boston subway network and the Internet. Nevertheless, their measure considers only geodesic information and does not capture information contained in network flows, the associated costs, and users' behavior, be it according to centralized or decentralized decision-making principles.

In this paper, we review the theoretical and practical foundations for the performance / efficiency measure proposed by Nagurney and Qiang (2007a-c, 2008a) that extends the Latora-Marchiori measure to incorporate such crucial network characteristics as decisionmaking induced flows and costs, in order to assess the importance of network components in a plethora of network systems. This network measure has significant advantages and captures the reality of networks today in that it explicitly considers congestion, which is a major problem of network systems from transportation to electric power ones. Furthermore, the measure can handle both fixed and elastic demand network problems (cf. Qiang and Nagurney (2008)) plus time-dependent, dynamic networks, of specific relevance to the Internet (see Nagurney, Parkes, and Daniele (2007)). In addition, the measure allows for the ranking of network components, that is, the nodes and links, or combinations thereof, in terms of their importance from an efficiency / performance standpoint. This has significant implications for planning, maintenance, and emergency and disaster preparedness purposes as well as for national security. Indeed, the topic of centrality of nodes (and that of links or "edges") in a network is a major issue in network characterization (cf. Barrat et al. (2004)) with contributors from sociology (cf. Freeman (1979), Bonacich (1972), and Freeman, Borgatti, and White (1991)) as well as physics (see Newman (2004), Barrat, Barthélémy, and Vespignani (2005), and Dall'Asta et al. (2006)). Nevertheless, decision-maker behavior, and the associated readjustment after nodal or link removal, is not captured in their centrality measures.

Moreover, in addition to quantifying and assessing the impact of the complete disruption / removal of a network component (or subset of components), we also address the concept of network robustness, another important aspect associated with network vulnerability. For example, network robustness is concerned with the reduction in network resources, such as link capacity. According to the Institute of Electrical and Electronics Engineers (1990), the robustness of a system is "the degree to which a system or component can function correctly in the presence of invalid inputs or stressful conditions." This topic is very relevant 
since the U.S. infrastructure is now experiencing tremendous aging and deterioration, which exposes the networks and populations to additional vulnerability. Over one quarter of the 590,750 bridges in the U.S. have been rated as structurally deficient or functionally obsolete. The degradation of transportation networks due to poor maintenance, natural disasters, deterioration over time, as well as unforeseen attacks, now leads, in the U.S., to estimates of $\$ 94$ billion in terms of needed repairs for roads alone. Poor road conditions in the U.S. cost motorists $\$ 54$ billion in repairs and operating costs annually (see U.S. Department of Transportation Federal Highway Administration (2004) and American Society of Civil Engineers (2005)).

In 2009, President Obama signed the American Recovery and Reinvestment Act (ARRA) with $\$ 132$ billion of the allocated $\$ 787$ billion to be for the transportation sector (see Sahadi (2009)). Without careful planning and assessment, however, such stimulus funds may not have the desired outcomes. Indeed, according to the Braess (1968) paradox it is well-known that, under user-optimizing behavior, the addition of a new road may result in all travelers being worse off in terms of travel cost/time (see also Braess, Nagurney, and Wakolbinger $(2005))$ ! In this paper, we review network robustness measures under different decisionmaking behaviors, with a focus on transportation, due to the generality of the associated theories and methodologies, as well as connections to other network systems.

This paper, therefore, also exploits established connections between transportation networks and different network systems (cf. Nagurney (2006b), Liu and Nagurney (2007, 2009), Nagurney, Parkes, and Daniele (2007), Wu et al. (2006)) and demonstrates how the unified network measure can be applied to financial networks as well as, in the dynamic context, to the Internet, and even to supply chain networks in the case of random demands. For example, in the case of supply chain networks, several recent major disruptions and the associated impacts have graphically demonstrated the need to address supply-side risk with a case in point being a fire in the Phillips Semiconductor plant in Albuquerque, New Mexico, causing its major customer, Ericsson, to lose $\$ 400$ million in potential revenues. On the other hand, another major customer, Nokia, managed to arrange alternative supplies and, therefore, mitigated the impact of the disruption (cf. Latour (2001)).

Another illustrative example concerns the impact of Hurricane Katrina, with the consequence that $10 \%$ - $15 \%$ of total US gasoline production was halted, which not only raised the oil price in the US, but also overseas (see, e. g., Canadian Competition Bureau (2006)). More recently, in April 2010, a volcano erupted in Iceland, and spewed ash for miles which created havoc and chaos not only for passenger air travel in Europe and beyond, but also for freight shipments, for days. This was the greatest disruption to air travel since 9/11. 
Affected companies, in dealing with this major disruption with cascading impacts, sought alternative routes and alternative modes of transportation for their products from their origins to their destinations, and affected air travelers, where feasible, did the same (cf. Werdigier, Cowell, and Clark (2010)).

As recognized by Sheffi (2005), one of the main characteristics of disruptions in supply networks is "the seemingly unrelated consequences and vulnerabilities stemming from global connectivity." Supply chain disruptions may have impacts that propagate not only locally but globally and, therefore, as also stressed by Qiang, Nagurney, and Dong (2009), a holistic, system-wide approach to supply chain network modeling and analysis is essential in order to be able to capture the complex interactions among decision-makers. The same holds for the assessment of financial network vulnerability, as evidenced by the financial credit crisis of 2008 and 2009, with the economic troubles in the US cascading through overseas markets and the financial landscape changed for forever (cf. Chari, Christiano, and Kehoe (2008) and Nagurney and Qiang (2008b)).

We then move forward from the topics of network vulnerability and robustness, which are of direct relevance to critical infrastructure (see also Murray and Grubesic (2007)), to the exploration of synergy associated with network systems. We focus on their integration in the context of applications ranging from corporate ones, as in mergers and acquisitions, to humanitarian ones, as in the case of teaming, partnering, and collaboration in logistics operations. As noted by Langabeer (2003) there were over 6,000 mergers and acquisitions (M\&As) transactions conducted world-wide in 2001, with a value of over a trillion dollars. Nevertheless, many scholars argue whether or not mergers achieve their objectives. For example, Marks and Mirvis (2001) found that fewer than $25 \%$ of all mergers achieve their stated objectives. Langabeer and Seifert (2003) determined a direct correlation between how effectively supply chains of merged firms are integrated and how successful the merger is. Furthermore, they state, based on the empirical findings in Langabeer (2003), who analyzed hundreds of mergers over the past decade, that improving supply chain integration between merging companies is the key to improving the likelihood of post-merger success.

We take, as a foundation, a system-optimization perspective for network integration, which is flexible and general enough to also be expandable to multiple products, multicriteria decision-making behavior, as well as to oligopolistic competition (cf. Nagurney (2008, 2009), Nagurney and Woolley (2010), and Nagurney, Woolley, and Qiang (2010)). Given the uncertain economic and financial climate, it is imperative to assess a priori the possible synergy (if any) associated with potential network integration, including those associated with mergers and acquisitions. According to Reuters (2010), the Organization for Economic 
Co-operation and Development (OECD) noted that the monthly average M\&A activity, in the year-to end February 2010, was worth just under $\$ 50$ billion, which is at the lowest level since the beginning of the global economic crisis.

In addition, we emphasize that a system-optimization framework for network integration may be used and applied also in the context of humanitarian operations and logistics. The supply chain network, for example, is a critical component not only of corporations but also of humanitarian organizations and their logistical operations. Humanitarian supply chains are more extended, fragile, and time-sensitive today than ever before. Moreover, the need to deliver vital goods (and services) to populations in times of crises is ever more pressing. The current humanitarian logistics environment requires that organizations mitigate risks and operate efficiently, which has spurred interest as to how to use supply chains for humanitarian logistics most efficiently and effectively. Therefore, how to organize and operate an effective humanitarian logistics network is of great value to practitioners and also of interest to researchers. This paper also overviews supply chain network models with nonlinear costs that can capture the reality of congestion, which may occur in humanitarian disaster relief operations. It is important to acknowledge the unique characteristics of humanitarian logistics operations in a supply chain context and how they differ from commercial supply chains. For example, Van Wassenhove (2006) delineates the differences between the environment surrounding disaster relief versus the commercial environment with major implications for the underlying supply chain networks. Clearly, the importance of the decisions as to what to offer in terms of products and services as well as the ability of humanitarian organizations to realize synergistic opportunities of integrated networks, can add tremendous value and, perhaps, even save lives.

This paper is organized as follows. In Section 2, we focus on the user-optimized or network equilibrium model(s) and present the unified network efficiency/performance measure. We also present the network component importance indicator based on the measure. Plus, we overview a network robustness measure under user-optimizing behavior, which quantifies the impact of link capacity degradation or investments resulting in link capacity enhancements. In addition, we highlight several applications and present some illustrative numerical examples. The outlined tools enable cognizant decision-makers and policy analysts to address such important questions as: Which are the most important roads and bridges in a region? Which banks, if they were to fail, would cause the greatest impact? Which production facilities for critical needs products, such as vaccines, are the most important? Which electric power stations are most critical?

In Section 3, we recall the classical system-optimized network model with separable total 
link cost functions, as well as a more general one, and explore concepts of network robustness from the perspective of relative total cost and under alternative decision-making behaviors. We also provide examples and identify the relationship between the relative total cost indices and the price of anarchy.

In Section 4, we overview network integration, with a focus on supply chains, and the associated synergies for the assessment of a wide spectrum of applications from mergers and acquisitions to humanitarian logistics operations. In the models in this Section, unlike those in Section 3, link capacities are explicitly imposed. In Section 5, we describe our recent research of relevance to network fragility and vulnerability from the perspective of network design and give suggestions for future research. 


\section{Decentralized Decision-Making and User-Optimizing Behavior}

In this Section and the next, we recall some fundamental network models based on distinct principles of decision-making behavior (due to Wardrop (1952)), which have come to be known, respectively, as user-optimizing (U-O) or system-optimizing (S-O) behavior (cf. Dafermos and Sparrow (1969)). These principles were originally cast in the context of transportation networks, in which travelers selected, in the case of U-O, their optimal routes of travel between origins and destinations so as to minimize their personal or user cost or, in the case of $\mathrm{S}-\mathrm{O}$, were routed in a manner so that the total cost to society was minimized. For background on these principles and their classical mathematical formulation due to Beckmann, McGuire, and Winsten (1956), see the overview by Boyce, Mahmassani, and Nagurney (2005). Another way of interpreting and understanding these alternative principles of network behavior is to consider user-optimizing behavior as being decentralized or "selfish" and system-optimizing behavior as being "unselfish" or centralized.

In this Section, we focus on user-optimizing behavior on networks. We present some fundamental models and overview the unified network efficiency / performance measure and the network component importance indicator. We also present a network robustness measure under user-optimizing behavior. In Section 3, we recall the system-optimizing model(s) and turn to the measurement of network robustness using relative total cost indices under either U-O or S-O behaviors.

User-optimized network models are also commonly referred to as network equilibrium models. We first recall in Section 2.1 the network equilibrium model with elastic demands with given inverse demand or disutility functions (see Dafermos (1982)). We then provide in Section 2.2 a special case in which the demands are assumed fixed and known. These models were originally proposed in the context of transportation but, given their wide applicability, the presentation below is for any network equilibrium problem. Indeed, Nagurney (2006b), Liu and Nagurney (2007), and Wu et al. (2006) have shown, respectively, that supply chain networks, financial networks, and electric power generation and distribution networks in which there are multiple interacting decision-makers, can be reformulated and solved as (transportation) network equilibrium problems over appropriately constructed abstract networks or supernetworks (Nagurney and Dong (2002)). In addition, (cf. Nagurney, Parkes, and Daniele (2007) and the references therein) the Internet also exhibits behavior similar to that of transportation network equilibrium problems, including the occurrence of the Braess (1968) paradox. 


\subsection{The Network Equilibrium Model with Elastic Demands}

We consider a network $\mathcal{G}=[\mathcal{N}, \mathcal{L}]$ consisting of the set of nodes $\mathcal{N}$ with $n$ elements and the set of directed links $\mathcal{L}$ with $K$ elements. The set of origin/destination (O/D) pairs of nodes is denoted by $W$ and has $n_{W}$ elements, and the set of acyclic paths joining the $\mathrm{O} / \mathrm{D}$ pairs is denoted by $P$ and has $n_{P}$ elements.

We denote the set of acyclic paths joining O/D pair $w$ by $P_{w}$. Links are denoted by $a, b$, etc; paths by $p, q$, etc., and $\mathrm{O} / \mathrm{D}$ pairs by $w_{1}, w_{2}$, etc.

We denote the nonnegative flow on path $p$ by $x_{p}$ and the flow on link $a$ by $f_{a}$ and we group the path flows into the vector $x \in R_{+}^{n_{P}}$ and the link flows into the vector $f \in R_{+}^{K}$. The link flows are related to the path flows through the following conservation of flow equations:

$$
f_{a}=\sum_{p \in P} x_{p} \delta_{a p}, \quad \forall a \in \mathcal{L}
$$

where $\delta_{a p}=1$ if link $a$ is contained in path $p$, and $\delta_{a p}=0$, otherwise. Hence, the flow on a link is equal to the sum of the flows on paths that contain that link.

The user cost on a path $p$ is denoted by $C_{p}$ and the user cost on a link $a$ by $c_{a}$. We denote the demand associated with $\mathrm{O} / \mathrm{D}$ pair $w$ by $d_{w}$ and the disutility by $\lambda_{w}$.

The user costs on paths are related to user costs on links through the following equations:

$$
C_{p}=\sum_{a \in \mathcal{L}} c_{a} \delta_{a p}, \quad \forall p \in P
$$

that is, the user cost on a path is equal to the sum of user costs on links that make up the path.

The user link cost function on each link may, in general, depend upon the entire vector of link flows so that

$$
c_{a}=c_{a}(f), \quad \forall a \in \mathcal{L} .
$$

We assume that the user link cost functions are continuous and monotonically increasing (to capture congestion).

The following conservation of flow equations must hold:

$$
\sum_{p \in P_{w}} x_{p}=d_{w}, \quad \forall w \in W
$$

which means that the sum of path flows on paths connecting each $\mathrm{O} / \mathrm{D}$ pair must be equal to the demand for that $\mathrm{O} / \mathrm{D}$ pair. 
The disutility (that is, the inverse demand) functions for the $\mathrm{O} / \mathrm{D}$ pairs are assumed as given and to be continuous and monotonically decreasing, where

$$
\lambda_{w}=\lambda_{w}(d), \quad \forall w \in W
$$

where $d$ is the vector of demands.

\section{Definition 1: Network Equilibrium - Elastic Demands}

A path flow and demand pattern $\left(x^{*}, d^{*}\right) \in \mathcal{K}^{1}$, where $\mathcal{K}^{1} \equiv\left\{(x, d) \mid(x, d) \in R_{+}^{n_{P}+n_{W}}\right.$ and (4) holds\}, is said to be a network equilibrium, in the case of elastic demands, if, once established, no user has any incentive to alter his "travel" decisions. This state is expressed by the following condition which must hold for each $O / D$ pair $w \in W$ and every path $p \in P_{w}$ :

$$
C_{p}\left(x^{*}\right) \begin{cases}=\lambda_{w}\left(d^{*}\right), & \text { if } \quad x_{p}^{*}>0 \\ \geq \lambda_{w}\left(d^{*}\right), & \text { if } \quad x_{p}^{*}=0\end{cases}
$$

Condition (6) states that all utilized paths connecting an O/D pair have equal and minimal user costs and these costs are equal to the disutility associated with using that O/D pair. As established in Dafermos (1982), the network equilibrium condition (6) is equivalent to the following variational inequality problem.

\section{Theorem 1}

A path flow and demand pattern $\left(x^{*}, d^{*}\right) \in \mathcal{K}^{1}$ is an equilibrium according to Definition 1 if and only if it satisfies the variational inequality: determine $\left(x^{*}, d^{*}\right) \in \mathcal{K}^{1}$ such that

$$
\sum_{w \in W} \sum_{p \in P_{w}} C_{p}\left(x^{*}\right) \times\left[x_{p}-x_{p}^{*}\right]-\sum_{w \in W} \lambda_{w}\left(d^{*}\right) \times\left[d_{w}-d_{w}^{*}\right] \geq 0, \quad \forall(x, d) \in \mathcal{K}^{1}
$$

\subsection{Network Equilibrium Model with Fixed Demands}

Assume now that the demands are fixed and known. We then have that Definition 1 simplifies to:

\section{Definition 2: Network Equilibrium - Fixed Demands}

A path flow pattern $x^{*} \in \mathcal{K}^{2}$, where $\mathcal{K}^{2} \equiv\left\{x \mid x \in R_{+}^{n_{P}}\right.$ and (4) holds with $d_{w}$ known and fixed for each $w \in W\}$, is said to be a network equilibrium, in the case of fixed demands, if the 
following condition holds for each $O / D$ pair $w \in W$ and each path $p \in P_{w}$ :

$$
C_{p}\left(x^{*}\right) \begin{cases}=\lambda_{w}, & \text { if } \quad x_{p}^{*}>0, \\ \geq \lambda_{w}, & \text { if } \quad x_{p}^{*}=0 .\end{cases}
$$

Condition (8) signifies that all used paths connecting an O/D pair have equal and minimal costs (see also Wardrop (1952) and Beckmann, McGuire, and Winsten (1956)). As proved in Smith (1979) and Dafermos (1980), the fixed demand network equilibrium condition (8) is equivalent to the following variational inequality problem.

\section{Theorem 2}

A path flow pattern $x^{*} \in \mathcal{K}^{2}$ is a network equilibrium according to Definition 2 if and only if it satisfies the variational inequality problem: determine $x^{*} \in \mathcal{K}^{2}$ such that

$$
\sum_{w \in W} \sum_{p \in P_{w}} C_{p}\left(x^{*}\right) \times\left[x_{p}-x_{p}^{*}\right] \geq 0, \quad \forall x \in \mathcal{K}^{2} .
$$

Obviously, (9) can be obtained directly from (7) by noting that, in the case of fixed demands, $d_{w}^{*}=d_{w}, \forall w \in W$.

Both variational inequalities (7) and (9) are in path flows. There are analogous variational inequalities, but in link flows (see, e.g., Nagurney and Qiang (2009a)). Moreover, in the case that the user link cost functions (3) are separable, in that the cost on a link depends only on the flow on that link, and the travel disutility functions (5) are also separable, then the elastic demand network equilibrium condition (6) (and, hence, variational inequality (7)) can be reformulated as the solution to the following convex optimization problem (see also Beckmann, McGuire, and Winsten (1956)):

$$
\text { Minimize } \sum_{a \in \mathcal{L}} \int_{0}^{f_{a}} c_{a}(y) d y-\sum_{w \in W} \int_{0}^{d_{w}} \lambda_{w}(z) d z,
$$

subject to: (1), (4), and $x_{p} \geq 0, \forall p \in P$. Similarly, in the case of the fixed demand model, the corresponding optimization reformulation of the fixed demand equilibrium condition (8) would have an objective function as above but with the second term removed and constraint (4) would be for fixed demands.

Existence of a solution to variational inequality (9) is guaranteed from the standard theory of variational inequalities (see e.g., Nagurney (1999)) under the assumption that the 
link cost functions and, hence, the path cost functions are continuous since the feasible set $\mathcal{K}^{2}$ is compact. Uniqueness of an equilibrium link flow pattern, in turn, is then guaranteed under the assumption that the user link cost functions are strictly monotone. In the case of variational inequality (7) stronger conditions need to be imposed to obtain existence of a solution. We note that, in particular, strong monotonicity of the link cost functions and minus the disutility functions will guarantee uniqueness of the corresponding equilibrium link flow and demand pattern (see also Nagurney (1999)). Algorithms for the solution of variational inequalities (7) and (9) can be found in Nagurney (1999), Nagurney and Zhang (1996), and the references therein. Algorithms, which have been widely applied in practice, for the solution of classical traffic network equilibrium problems, which can be reformulated as optimization problems, can be found in Patriksson (1994), Bar-Gera (2002), Boyce, Mahmassani, and Nagurney (2005), Nagurney and Qiang (2009a), and the references therein.

It is clear that an appropriate and unified network performance/efficiency measure should be suitable for networks with either elastic demands or fixed demands.

\subsection{A Unified Network Performance Measure}

Before we recall the unified network performance measure of Qiang and Nagurney (2008) (see also Nagurney and Qiang (2007a-c)) we review an important property that such a measure should have.

\section{Network Performance Property:}

The performance/efficiency measure for a given network should be nonincreasing with respect to the equilibrium disutility for each $O / D$ pair, holding the equilibrium disutilities for the other $O / D$ pairs constant.

Given this desirable property of a network performance measure, the unified network performance measure is as below.

\section{Definition 3: A Unified Network Performance Measure}

The network performance/efficiency measure, $\mathcal{E}(\mathcal{G}, d)$, for a given network topology $\mathcal{G}$ and the equilibrium (or fixed) demand vector $d$, is:

$$
\mathcal{E}=\mathcal{E}(\mathcal{G}, d)=\frac{\sum_{w \in W} \frac{d_{w}}{\lambda_{w}}}{n_{W}},
$$

where recall that $n_{W}$ is the number of $O / D$ pairs in the network, and $d_{w}$ and $\lambda_{w}$ denote, for 
simplicity, the equilibrium (or fixed) demand and the equilibrium disutility for $O / D$ pair w, respectively.

According to $\mathcal{E}$, the elimination of a link is treated by removing that link from the network while the removal of a node is managed by removing the links entering and exiting that node. If the removal results in no path connecting an $\mathrm{O} / \mathrm{D}$ pair, we just assign the demand for that $\mathrm{O} / \mathrm{D}$ pair (either fixed or elastic) to an abstract path at a cost of infinity.

Interestingly, as established in Qiang and Nagurney (2008), under certain assumptions, the unified measure collapses to the Latora and Marchiori (2001) measure, which, however, considers neither explicit demands nor flows and is as follows:

\section{Definition 4: The Latora and Marchiori Measure}

Let $n$ be the number of nodes in $\mathcal{G}$. Then the Latora and Marchiori network efficiency measure $E$ is:

$$
E=E(\mathcal{G})=\frac{1}{n(n-1)} \sum_{i \neq j \in \mathcal{G}} \frac{1}{d_{i j}},
$$

where $d_{i j}$ is the shortest path length (geodesic distance) between nodes $i$ and $j$.

\section{Theorem 3}

If positive demands exist for all pairs of nodes in the network $\mathcal{G}$, and each of these demands is equal to 1 and if $d_{i j}$ is set equal to $\lambda_{w}$, where $w=(i, j)$, for all $w \in W$ then the proposed network efficiency measure $\mathcal{E}$ and the $E$ measure are one and the same.

The proof of the above theorem assumes that $d_{i j}$ is equal to the corresponding $\lambda_{w}$, which is not unreasonable. The $\mathcal{E}$ measure, however, is more general since it captures the flows on networks and their reallocation, in the case of disruptions, through the demands, disutilities, and costs.

For a network with fixed demands, it is easy to verify that the unified measure $\mathcal{E}$ is welldefined. In a network with elastic demands, when there is a disconnected O/D pair $w$, we have, from the above discussion, that the associated "path cost" of the abstract path, say, $r, C_{r}\left(x^{*}\right)$, is equal to infinity. If the disutility functions are known, according to equilibrium condition (6), we then have that $C_{r}\left(x^{*}\right)>\lambda_{w}\left(d^{*}\right)$, and, hence, $x_{r}^{*}=0$, so that $d_{w}^{*}=0$, which leads to the conclusion of $d_{w}^{*} / \lambda_{w}=0$. Therefore, the disconnected O/D pair $w$ makes zero "contribution" to the efficiency measure and $\mathcal{E}$ is well-defined in both the fixed and elastic demand cases. We can expect a network to get disconnected in the case of disasters and, consequently, our measure has the essential feature that it is well-defined even in such 
situations.

The unified measure $\mathcal{E}$ has the following interpretation in the case of transportation networks. The equilibrium $\mathrm{O} / \mathrm{D}$ pair disutility, $\lambda_{w}$, is proportional to the (travel) time between each O/D pair $w \cdot d_{w}$ is the equilibrium demand (in terms of total vehicles) between each O/D pair $w$. Therefore, $d_{w} / \lambda_{w}$ is the (vehicle) throughput between $\mathrm{O} / \mathrm{D}$ pair $w \cdot \mathcal{E}(\mathcal{G}, d)$ is the average (vehicle) throughput on the network $\mathcal{G}$ with demand vector $d$. The higher the throughput that a network has, the better its performance and the more efficient it is. For general networks, $\mathcal{E}$ is actually the average demand to price ratio. When $\mathcal{G}$ and $d$ are fixed, a network is more efficient if it can satisfy a higher demand at a lower price.

\subsection{The Importance of Network Components}

With the network performance/efficiency measure, we are ready to investigate the importance of network components by studying their impact on the network efficiency through their removal. The network efficiency can be expected to deteriorate when a critical network component is eliminated from the network. Such a component can include a link or a node or a subset of nodes and links depending on the network problem under investigation. We expect that the removal of a critical network component will cause greater impact than that of a trivial one. Hence, the importance of a network component is defined, following Qiang and Nagurney (2008), as follows.

\section{Definition 5: Importance of a Network Component}

The importance of a network component $g \in \mathcal{G}, I(g)$, is measured by the relative network efficiency drop after $g$ is removed from the network:

$$
I(g)=\frac{\triangle \mathcal{E}}{\mathcal{E}}=\frac{\mathcal{E}(\mathcal{G}, d)-\mathcal{E}(\mathcal{G}-g, d)}{\mathcal{E}(\mathcal{G}, d)}
$$

where $\mathcal{G}-g$ is the resulting network after component $g$ is removed from network $\mathcal{G}$.

The upper bound of the importance of a network component is 1 . The higher the value, the more important a network component is.

We now present an example to illustrate $\mathcal{E}$ and $E$ and provide a discussion. We then apply both measures to the Braess paradox (1968) network. Note that Latora and Marchiori (2004) defined the importance of a network component as $I(g)=E(\mathcal{G})-E(\mathcal{G}-g)=\Delta E$,

but they use $I(g)=\frac{\Delta E}{E}$ in their calculations, which we do as well when we compare the $\mathcal{E}$ measure to the $E$ measure. 


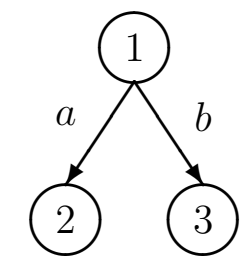

Figure 1: Example 1

Table 1: Importance Values and Ranking of Links in Example 1

\begin{tabular}{|c|c|c|c|c|}
\hline Link & $\begin{array}{c}\text { Importance Value } \\
\text { from } \mathcal{E}\end{array}$ & $\begin{array}{c}\text { Importance Ranking } \\
\text { from } \mathcal{E}\end{array}$ & $\begin{array}{c}\text { Importance Value } \\
\text { from } E\end{array}$ & $\begin{array}{c}\text { Importance Ranking } \\
\text { from } E\end{array}$ \\
\hline$a$ & 0.8333 & 1 & 0.5000 & 1 \\
\hline$b$ & 0.1667 & 2 & 0.5000 & 1 \\
\hline
\end{tabular}

\section{An Example}

Consider the network in Figure 1 in which there are two O/D pairs: $w_{1}=(1,2)$ and $w_{2}=$ $(1,3)$ with demands given, respectively, by $d_{w_{1}}=100$ and $d_{w_{2}}=20$. We have that path $p_{1}=a$ and path $p_{2}=b$. Assume that the link cost functions are given by: $c_{a}\left(f_{a}\right)=$ $.01 f_{a}+19$ and $c_{b}\left(f_{b}\right)=.05 f_{b}+19$. Clearly, we must have that $x_{p_{1}}^{*}=100$ and $x_{p_{2}}^{*}=20$ so that $\lambda_{w_{1}}=\lambda_{w_{2}}=20$. The network efficiency measure $\mathcal{E}=\frac{1}{2}\left(\frac{100}{20}+\frac{20}{20}\right)=3.0000$ whereas $E=\frac{1}{6}\left(\frac{1}{20}+\frac{1}{20}\right)=.0167$.

The importance values and the rankings of the links and the nodes for Example 1 are given, respectively, in Tables 1 and 2, using the importance measures $\mathcal{E}$ and $E$.

Clearly, $\mathcal{E}$, which captures flow information, is the more general, reasonable, and precise measure, since, in the case of a disruption, the destruction of link $a$, with which was associated a flow 5 times the flow of link $b$, would result in a greater loss of efficiency. The same

Table 2: Importance Values and Ranking of Nodes in Example 1

\begin{tabular}{|c|c|c|c|c|}
\hline Node & $\begin{array}{c}\text { Importance Value } \\
\text { from } \mathcal{E}\end{array}$ & $\begin{array}{c}\text { Importance Ranking } \\
\text { from } \mathcal{E}\end{array}$ & $\begin{array}{c}\text { Importance Value } \\
\text { from } E\end{array}$ & $\begin{array}{c}\text { Importance Ranking } \\
\text { from } E\end{array}$ \\
\hline 1 & 1.0000 & 1 & 1.0000 & 1 \\
\hline 2 & 0.8333 & 2 & 0.5000 & 2 \\
\hline 3 & 0.1667 & 3 & 0.5000 & 2 \\
\hline
\end{tabular}



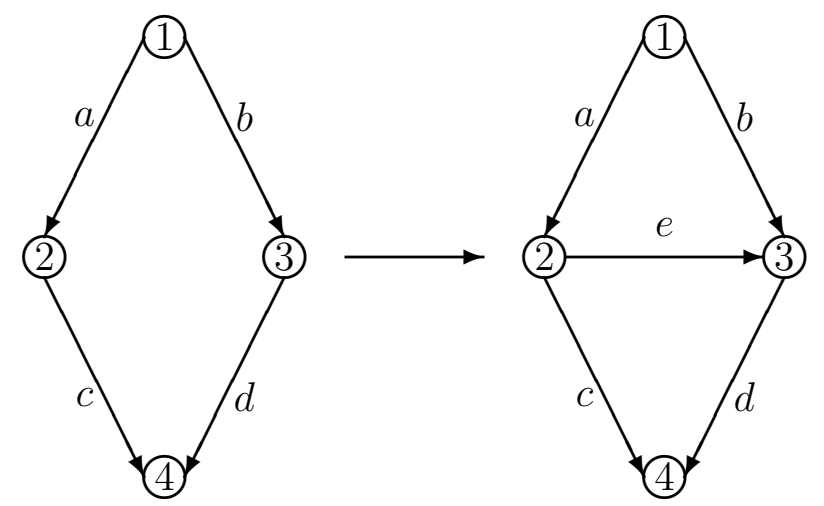

Figure 2: The Braess Network Example

qualitative analysis holds for the destruction of node 2 versus node 3.

\subsection{An Application to the Braess Paradox}

In order to further reinforce the above concepts, we now recall the well-known Braess (1968) paradox; see also Braess, Nagurney, and Wakolbinger (2005). This paradox is as relevant to transportation networks as it is to telecommunication networks, and, in particular, to the Internet, since such networks are subject to traffic operating in a decentralized decisionmaking manner (cf. Korilis, Lazar, and Orda (1999), Nagurney, Parkes, and Daniele (2007), and the references therein).

Assume a network as the first network depicted in Figure 2 in which there are four nodes: $1,2,3,4$; four links: $a, b, c, d$; and a single $\mathrm{O} / \mathrm{D}$ pair $w=(1,4)$. There are, hence, two paths available to travelers between this $\mathrm{O} / \mathrm{D}$ pair: $p_{1}=(a, c)$ and $p_{2}=(b, d)$.

The user link travel cost functions are:

$$
c_{a}\left(f_{a}\right)=10 f_{a}, \quad c_{b}\left(f_{b}\right)=f_{b}+50, \quad c_{c}\left(f_{c}\right)=f_{c}+50, \quad c_{d}\left(f_{d}\right)=10 f_{d} .
$$

Assume a fixed travel demand $d_{w}=6$.

It is easy to verify that the equilibrium path flows are: $x_{p_{1}}^{*}=3, x_{p_{2}}^{*}=3$, the equilibrium link flows are: $f_{a}^{*}=3, \quad f_{b}^{*}=3, f_{c}^{*}=3, \quad f_{d}^{*}=3$, with associated equilibrium path travel costs: $C_{p_{1}}=c_{a}+c_{c}=83, C_{p_{2}}=c_{b}+c_{d}=83$.

Assume now that, as depicted in Figure 2, a new link "e", joining node 2 to node 3 is added to the original network, with user link cost function $c_{e}\left(f_{e}\right)=f_{e}+10$. The addition of this link creates a new path $p_{3}=(a, e, d)$ that is available to the travelers. Assume that the travel demand $d_{w}$ remains at 6 units of flow. Note that the original flow distribution pattern 
Table 3: Link Results for the Braess Network

\begin{tabular}{|c|c|c|c|c|}
\hline \multirow{2}{*}{ Link } & \multicolumn{2}{|c|}{$\mathcal{E}$ Measure } & \multicolumn{2}{c|}{ E Measure } \\
\cline { 2 - 5 } & $\begin{array}{c}\text { Vmportance } \\
\text { Value }\end{array}$ & $\begin{array}{c}\text { Importance } \\
\text { Ranking }\end{array}$ & $\begin{array}{c}\text { Importance } \\
\text { Value }\end{array}$ & $\begin{array}{c}\text { Importance } \\
\text { Ranking }\end{array}$ \\
\hline$a$ & .2069 & 1 & .1056 & 3 \\
\hline$b$ & .1794 & 2 & .2153 & 2 \\
\hline$c$ & .1794 & 2 & .2153 & 2 \\
\hline$d$ & .2069 & 1 & .1056 & 3 \\
\hline$e$ & -.1084 & 3 & .3616 & 1 \\
\hline
\end{tabular}

Table 4: Nodal Results for the Braess Network

\begin{tabular}{|c|c|c|c|c|}
\hline \multirow[b]{2}{*}{ Node } & \multicolumn{2}{|c|}{$\mathcal{E}$ Measure } & \multicolumn{2}{|c|}{ E Measure } \\
\hline & $\begin{array}{l}\text { Importance } \\
\text { Value }\end{array}$ & $\begin{array}{c}\text { Importance } \\
\text { Ranking }\end{array}$ & $\begin{array}{l}\text { Importance } \\
\text { Value }\end{array}$ & $\begin{array}{c}\text { Importance } \\
\text { Ranking }\end{array}$ \\
\hline 1 & 1.0000 & 1 & - & - \\
\hline 2 & .2069 & 2 & .7635 & 1 \\
\hline 3 & .2069 & 2 & .7635 & 1 \\
\hline 4 & 1.0000 & 1 & - & - \\
\hline
\end{tabular}

$x_{p_{1}}=3$ and $x_{p_{2}}=3$ is no longer an equilibrium pattern, since at this level of flow the user cost on path $p_{3}, C_{p_{3}}=c_{a}+c_{e}+c_{d}=70$, so travelers would switch paths

The equilibrium flow pattern on the new network is: $x_{p_{1}}^{*}=2, x_{p_{2}}^{*}=2, x_{p_{3}}^{*}=2$, with equilibrium link flows: $f_{a}^{*}=4, f_{b}^{*}=2, f_{c}^{*}=2, f_{e}^{*}=2, f_{d}^{*}=4$, and with associated equilibrium user path travel costs: $C_{p_{1}}=C_{p_{2}}=C_{p_{3}}=92$. Note that the travel cost increased for every user of the network from 83 to 92 without a change in the travel demand!

We now apply the unified network efficiency measure $\mathcal{E}$ to the Braess network with the link $e$ to identify the importance and ranking of nodes and links. We also, for completeness, apply the $E$ measure. The results are reported in Tables 3 and 4 .

It is interesting that the links identified as the most important ones according to our measure $\mathcal{E}$, that is, links $a$ and $d$, are ranked the least important according to the $E$ measure. On the other hand, link $e$, which is ranked least important according to the $\mathcal{E}$ measure, is ranked as most important according to the $E$ measure. Because the addition of link $e$ causes the Braess paradox when demand is equal to 6, it will, obviously, be detrimental to network performance, which is clearly shown by the negative importance value of link $e$ obtained via 
the $\mathcal{E}$ measure. The fact that link $e$ is ranked as the most important link according to the $E$ measure is unreasonable.

Moreover, note that in the above link and node ranking results the importance values (and hence their rankings) of nodes 1 and 4 are not defined for the $E$ measure. This is due to the fact that the cost functions on links $a$ and $d$ are solely dependent on the flow on the respective link (and do not have any fixed cost terms). Take node 1, for example, the removal of node 1 is treated by removing links $a$ and $b$. But the cost on link $d$ will be zero because of the cost structure on the link, which makes the $E$ not defined. However, our measure $\mathcal{E}$ is still well-defined with the removal of nodes 1 and 4 .

$\mathcal{E}$ has been applied to rank the importance of network components in several real-world transportation networks including the Anaheim, California network and the Sioux Falls, Dakota network (see Nagurney and Qiang (2009a)). In addition, it has been applied by Schulz (2007) to evaluate highways in Germany and found to outperform several existing measures. It is worth mentioning that one can utilize the importance indicator (12) to assess

additions to a network in terms of the improvement of network efficiency/performance. For example, $\mathcal{E}$ has been applied to determine how efficient the proposed North Dublin metro would be by Walsh (2009).

Jenelius, Petersen, and Mattsson (2006) proposed several link importance indicators and applied them to the road network in northern Sweden, but they depend upon whether or not the $\mathrm{O} / \mathrm{D}$ pairs become disconnected. Other vulnerability indices, with a focus on transportation, have been proposed by Murray-Tuite and Mahmassani (2004) and by Taylor, Sekhar, and D'Este (2006).

\subsection{Network Robustness Under User-Optimizing Behavior}

We now recall the robustness measure of a network under user-optimizing or decentralized decision-making behavior due to Nagurney and Qiang (2007b). Here we consider networks with user link cost functions in which the capacities on the links are incorporated explicitly into the functional forms. Such functions include the well-known Bureau of Public Roads (BPR) (1964) functions, and the Davidson (1966) function as well as the M/M/1 delay function (Bertsekas and Gallager (1987) and Roughgarden (2005)) with the latter two used in telecommunications and the former in transportation. 


\section{Definition 6: Network Robustness Measure Under User-Optimizing Decision- Making Behavior}

The robustness measure $\mathcal{R}^{\gamma}$ for a network $\mathcal{G}$ with the vector of user link cost functions $c$, the vector of link capacities $u$, the vector of demands d (either fixed or elastic) is defined as the relative performance retained under a given uniform capacity retention ratio $\gamma$ with $\gamma \in(0,1]$ so that the new capacities are given by $\gamma u$. Its mathematical definition is

$$
\mathcal{R}^{\gamma}=\mathcal{R}(\mathcal{G}, c, \gamma, u)=\frac{\mathcal{E}^{\gamma}}{\mathcal{E}} \times 100 \%
$$

where $\mathcal{E}$ and $\mathcal{E}^{\gamma}$ are the network performance measures with the original capacities and the remaining capacities, respectively.

For example, if $\gamma=.8$, this means that the user link cost functions now have the link capacities given by $.8 u_{a}$ for all links $a \in \mathcal{L}$; if $\gamma=.4$, then the link capacities become $.4 u_{a}$ for all links $a \in \mathcal{L}$, and so on.

According to Definition 6, a network under a given level of capacity retention or deterioration is considered to be robust if the network performance stays close to the original level.

\section{Remark 1}

We can also study network robustness from the perspective of network capacity enhancement. Such an analysis provides insights into link investments. In this case $\gamma \geq 1$ and, for definiteness (and as suggested in Nagurney and Qiang (2009a)), we refer to the network robustness measure in this context as the "capacity increment ration."

Next, we recall the BPR functional form, for completeness, and easy reference.

\section{Bureau of Public Roads (BPR) Function}

The functional form of the Bureau of Public Roads (1964) link cost functions is

$$
c_{a}\left(f_{a}\right)=t_{a}^{0}\left[1+k\left(\frac{f_{a}}{u_{a}}\right)^{\beta}\right], \quad \forall a \in \mathcal{L},
$$

where $f_{a}$ is the flow on link $a ; u_{a}$ is the "practical" capacity on link $a$, which also has the interpretation of the level-of-service flow rate; $t_{a}^{0}$ is the free-flow travel time or cost on link

$a ; k$ and $\beta$ are the model parameters and both take on positive values. Often in applications $k=.15$ and $\beta=4$. In this context $c_{a}\left(f_{a}\right)$ measures the travel time on the link. 


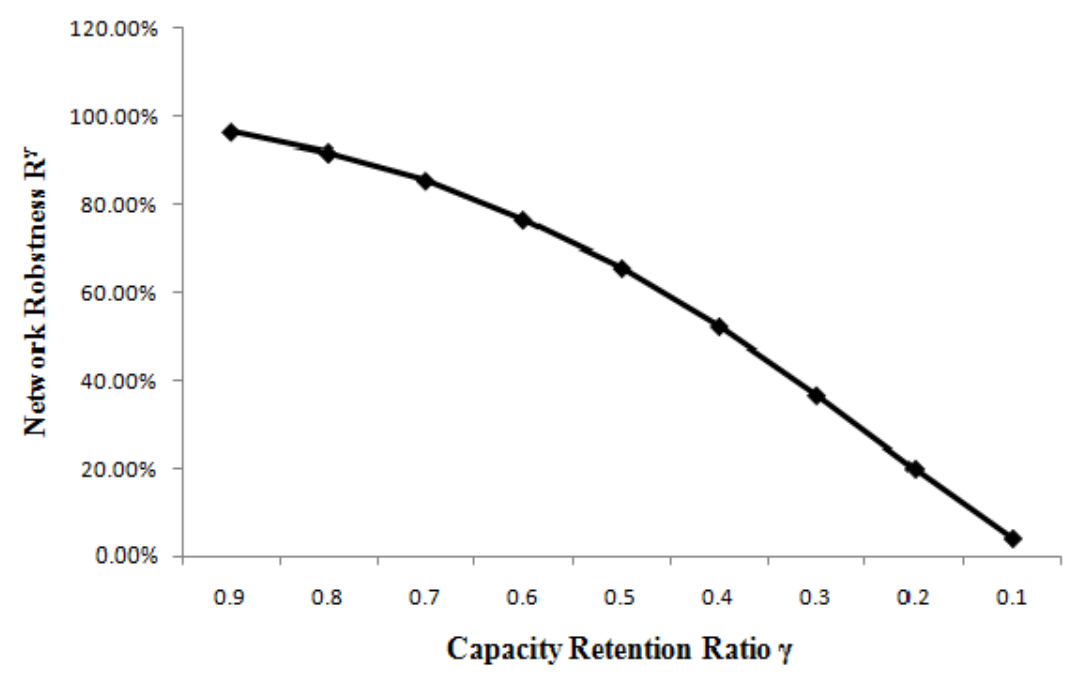

Figure 3: Robustness vs. Capacity Retention Ratio for the Anaheim Network

\subsection{An Application to the Anaheim Network}

In this Section, we illustrate the robustness measure (13) with an application to the Anaheim network. Each link of the Anaheim network has a link travel cost functional form of the BPR form (14). There are 461 nodes, 914 links, and 1, 406 O/D pairs in the Anaheim network. The relevant data and parameters in the BPR link cost functions are from the transportation network datasets maintained by Bar-Gera (2008) (http://www.bgu.ac.il/ bargera/tntp/). We used the projection method with the embedded equilibration algorithm (see Nagurney (1999)) and the column generation algorithm (cf. Leventhal, Nemhauser, and Trotter (1973)) to compute the equilibrium solutions. Then, based on these solutions, the network efficiency according to (10) and the importance values and the importance rankings of the links according to (12) were determined. The above computation schemes were implemented in MATLAB (www.mathworks.com) on an IBM T61 computer. The computed network efficiency measure for the Anaheim network is $\mathcal{E}=7.3651$. Additional results can be found in Nagurney and Qiang (2009a).

The results for different capacity retention ratios are displayed in Figure 3. Figure 4 presents the robustness of the Anaheim network with capacity enhancement (cf. Remark 1). We see that the robustness of the Anaheim network keeps increasing smoothly except for 


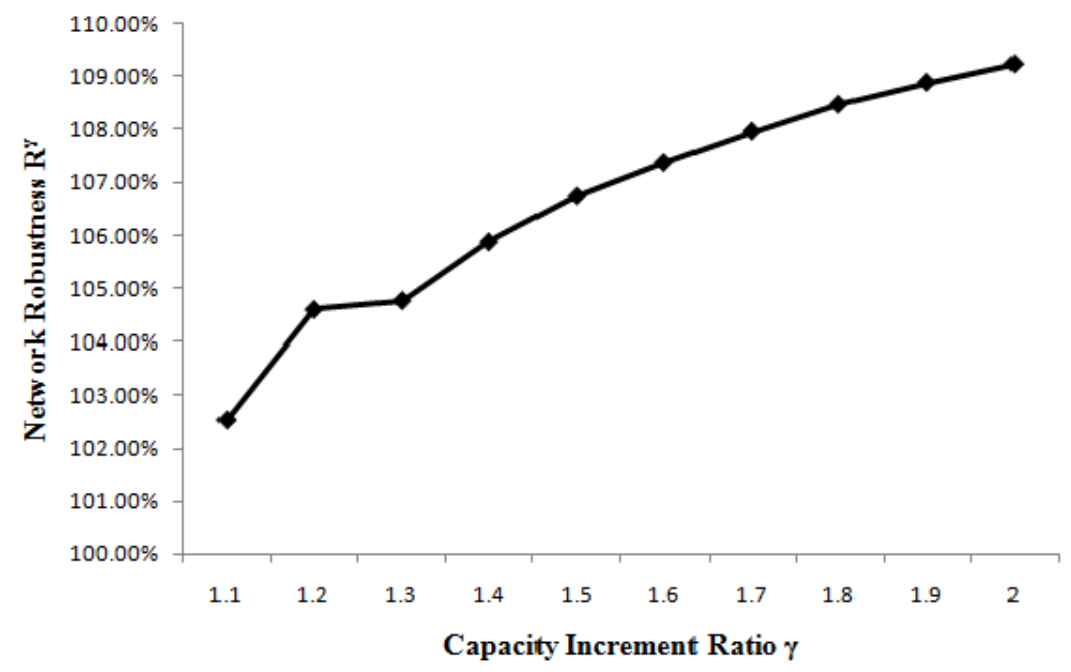

Figure 4: Robustness vs. Capacity Increment Ratio for the Anaheim Network

when $\gamma$ is in the range 1.2 and 1.3 , where the robustness increment is "flat."

\subsection{Network Efficiency / Performance Measures for Other Network Systems}

Qiang, Nagurney, and Dong (2009) developed a supply chain network equilibrium model with disruption risks and random demands with a general multitiered and multimodal structure as depicted in Figure 5. They proposed a supply chain network performance measure, which is adapted from (10), and applied it to several supply chains.

\section{Definition 7: The Supply Chain Network Performance Measure}

The supply chain network performance measure, $\mathcal{E}^{S C N}$, for a given supply chain network with topology $\mathcal{G}$ as in Figure 6 and expected demands: $\hat{d}_{k} ; k=1,2, \ldots, o$, is defined as follows

$$
\mathcal{E}^{S C N} \equiv \frac{\sum_{k=1}^{o} \frac{\hat{d}_{k}}{\rho_{3 k}}}{O}
$$

where $o$ is the number of demand markets in the supply chain network, and $\hat{d}_{k}$ and $\rho_{3 k}$ denote, respectively, the expected equilibrium demand and the equilibrium price at demand market $k$. 


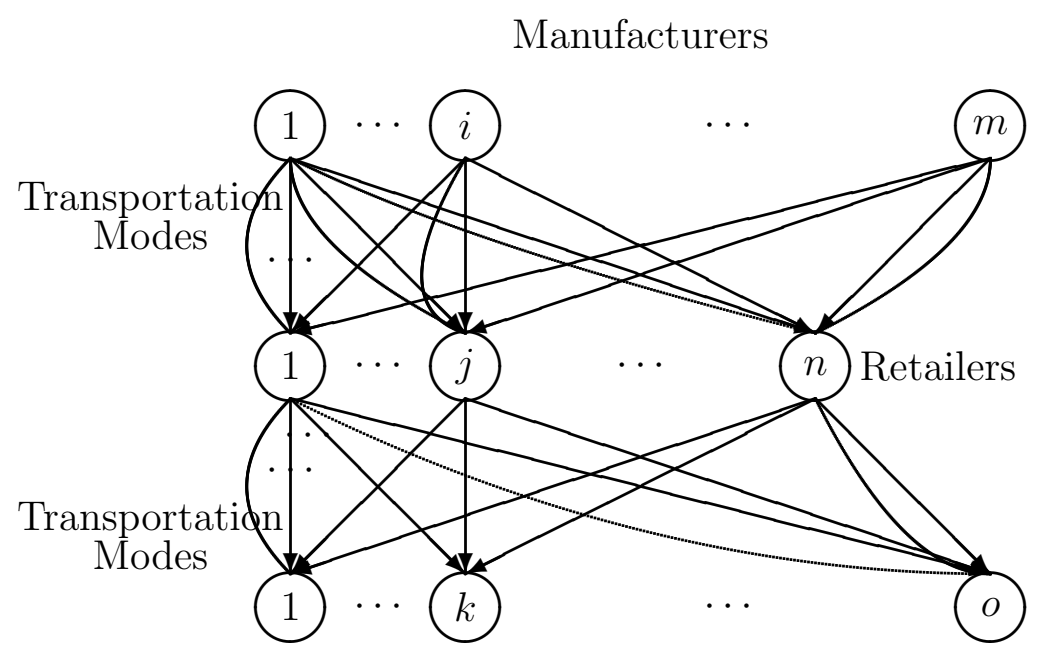

Demand Markets

Figure 5: The Supply Chain Network

Nagurney and Qiang (2008b) considered financial networks with intermediation and electronic transactions, originated by Nagurney and Ke (2003), with a structure as in Figure 6. They proposed the following performance measure which captures, in a holistic manner, the network interactions among the financial decision-makers.

\section{Definition 8: The Financial Network Performance Measure}

The financial network performance measure, $\mathcal{E}^{F N}$, for a given network topology $\mathcal{G}$ (cf. Figure $6)$, and demand price functions $\rho_{3 k}(d) ; k=1,2, \ldots, o$, and available funds held by the source agents, where $S$ is the vector of financial funds held by the source agents, is defined as follows

$$
\mathcal{E}^{F N}=\frac{\sum_{k=1}^{o} \frac{d_{k}^{*}}{\rho_{3 k}\left(d^{*}\right)}}{O},
$$

where $o$ is the number of demand markets in the financial network, and $d_{k}^{*}$ and $\rho_{3 k}\left(d^{*}\right)$ denote the equilibrium demand and the equilibrium price for demand market $k$, respectively.

The financial network performance measure $\mathcal{E}^{F N}(16)$ is the average demand to price ratio. It quantifiably captures the overall (economic) functionality of the financial network. When the network topology $\mathcal{G}$, the demand price functions, and the available funds held by source agents are given, a financial network is considered to be performing better if it can satisfy higher demands at lower prices. As shown in Nagurney and Qiang (2008b) the importance of financial network components is determined through a definition analogous to that of Definition 5. 
Sources of Financial Funds

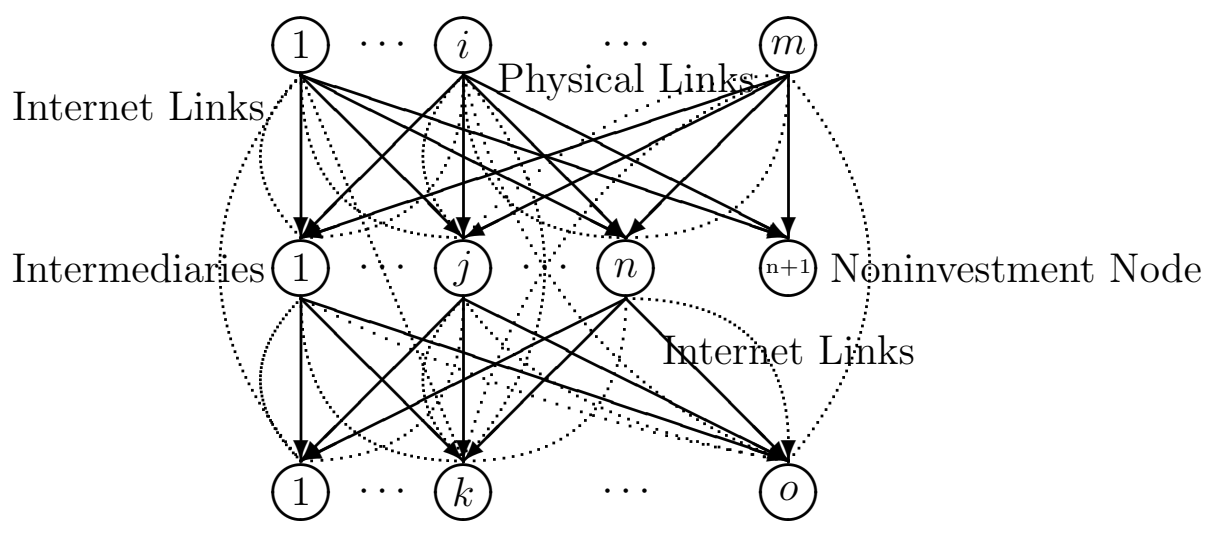

Demand Markets - Uses of Funds

Figure 6: The Financial Network with Intermediation and Electronic Transactions

\subsection{Efficiency Measurement of Dynamic Networks}

Nagurney and Qiang (2008c) proposed measures for dynamic networks, which are in continuous time and in discrete time, and which are applicable to the Internet and electric power networks. They also provided the definition of the importance of a dynamic network component, based on the measures. The framework was based on the dynamic network model of Nagurney, Parkes, and Daniele (2007), which allowed for the extension of the network equilibrium conditions (6) to the time-varying domain and made use of evolutionary (rather than finite-dimensional) variational inequalities. Specifically, they considered a time horizon $T$ and proposed the following efficiency measures.

\section{Definition 9: Dynamic Network Efficiency: Continuous Time Version}

The network efficiency for the network $\mathcal{G}$ with time-varying demand $d$ for $t \in[0, T]$, denoted by $\mathcal{E}^{D N}(\mathcal{G}, d, T)$, is defined as follows

$$
\mathcal{E}^{D N}(\mathcal{G}, d, T)=\frac{\int_{0}^{T}\left[\sum_{w \in W} \frac{d_{w}(t)}{\lambda_{w}(t)}\right] / n_{W} d t}{T} .
$$

The dynamic network efficiency measure $\mathcal{E}^{D N}$ is the average demand to price ratio over time. It measures the overall (economic) functionality of the network with time-varying demands. When the network topology $\mathcal{G}$, the demand pattern over time, and the time span are given, a network is considered to be more efficient if it can satisfy higher demands at lower costs over time. We assume that the integral in (17) is well-defined. 
The network efficiency measure (17) can be adapted to dynamic networks in which the demands change at discrete points in time. Let $d_{w}^{1}, d_{w}^{2}, \ldots, d_{w}^{H}$ denote demands for O/D pair $w$ in $H$ discrete time intervals, given, respectively, by: $\left[t_{0}, t_{1}\right],\left(t_{1}, t_{2}\right], \ldots,\left(t_{H-1}, t_{H}\right]$, where $t_{H} \equiv T$. We assume that the demand is constant in each such time interval for each $\mathrm{O} / \mathrm{D}$ pair. Moreover, we denote the corresponding minimal costs for each $\mathrm{O} / \mathrm{D}$ pair $w$ at the $H$ different time intervals by: $\lambda_{w}^{1}, \lambda_{w}^{2}, \ldots, \lambda_{w}^{H}$. The demand vector $d$, in this special discrete case, is a vector in $R^{n_{W} \times H}$. The dynamic network efficiency measure in this case is as follows.

\section{Definition 10: Dynamic Network Efficiency: Discrete Time Version}

The network efficiency for the network $(\mathcal{G}, d)$ over $H$ discrete time intervals: $\left[t_{0}, t_{1}\right],\left(t_{1}, t_{2}\right], \ldots,\left(t_{H-1}, t_{H}\right]$, where $t_{H} \equiv T$, and with the respective constant demands: $d_{w}^{1}, d_{w}^{2}, \ldots, d_{w}^{H}$ for all $w \in W$ is defined as

$$
\mathcal{E}^{D N}\left(\mathcal{G}, d, t_{H}=T\right)=\frac{\sum_{i=1}^{H}\left[\left(\sum_{w \in W} \frac{d_{w}^{i}}{\lambda_{w}^{i}}\right)\left(t_{i}-t_{i-1}\right) / n_{W}\right]}{t_{H}} .
$$

The relationship between the dynamic network efficiency measure (17) and the unified network efficiency measure (10) for static networks is given in the following theorem, due to Nagurney and Qiang (2008c).

\section{Theorem 4}

Assume that $d_{w}(t)=d_{w}$, for all $O / D$ pairs $w \in W$, and for $t \in[0, T]$. Then the dynamic network efficiency measure (17) collapses to the network measure $\mathcal{E}$ given by (10).

\section{Centralized Decision-Making and System-Optimizing Behavior}

We now consider centralized decision-making where a central controller seeks to determine the optimal flows between origin/destination pairs of nodes so as to satisfy the demands, but at minimal total cost. In such problems there is a single objective function to be optimized, subject to the constraints. Applications of the system-optimized (S-O) problem occur not only in transportation and telecommunication but also in a variety of settings, ranging from military operations to centralized supply chains, and even in the context of humanitarian logistics.

\subsection{The System-Optimized Problem}

As in Section 2, we assume, as given, the network $\mathcal{G}=[\mathcal{N}, \mathcal{L}]$, the user link cost functions, 
and the O/D pair demands, which are assumed to be fixed (and not elastic). We first focus on the S-O problem based on separable user link cost functions, that is, $c_{a}=c_{a}\left(f_{a}\right), \forall a \in \mathcal{L}$ and then consider more general user link cost functions and the associated total cost functions. These classical models are due, respectively, to Dafermos and Sparrow (1969) and Dafermos (1971).

The total cost on link $a$, denoted by $\hat{c}_{a}\left(f_{a}\right)$, is given by:

$$
\hat{c}_{a}\left(f_{a}\right)=c_{a}\left(f_{a}\right) \times f_{a}, \forall a \in \mathcal{L},
$$

since the total cost on a link is equal to the user link cost on the link times the flow on the link. The total cost on the network is, thus, expressed as

$$
\sum_{a \in \mathcal{L}} \hat{c}_{a}\left(f_{a}\right)
$$

The S-O problem is, hence:

$$
\text { Minimize } \sum_{a \in \mathcal{L}} \hat{c}_{a}\left(f_{a}\right),
$$

subject to: constraints (1), (4), and $x_{p} \geq 0, \forall p \in P$.

An alternative version of the S-O problem can be stated in path flow variables only:

$$
\text { Minimize } \sum_{p \in P} C_{p}(x) x_{p}
$$

subject to (4) and the nonnegativity assumption on the path flows, where the total cost on a path, denoted by $\hat{C}_{p}$, is the user cost on a path times the flow on a path, that is,

$$
\hat{C}_{p}=C_{p} x_{p}, \forall p \in P
$$

where recall that the user cost on a path, $C_{p}$, is given by (2).

\section{System-Optimality Conditions}

Under the assumption of convex (strictly convex) cost functions, the objective function (20) in the S-O problem is convex (strictly convex). The feasible set defined by constraint (1), (4), and the nonnegativity assumption on the path flows is also convex. Therefore, the optimality conditions, that is, the Kuhn-Tucker conditions (see Dafermos and Sparrow (1969)) are: for each $\mathrm{O} / \mathrm{D}$ pair $w \in W$ and each path $p \in P_{w}$, the nonnegative path flow pattern $x$ (and the

corresponding link flow pattern $f$ ), satisfying constraints (1) and (4), must satisfy:

$$
\hat{C}_{p}^{\prime}(x) \begin{cases}=\mu_{w}, & \text { if } \quad x_{p}>0 \\ \geq \mu_{w}, & \text { if } \quad x_{p}=0\end{cases}
$$


where $\hat{C}_{p}^{\prime}(x)$ denotes the marginal of the total cost on path $p$, given by:

$$
\hat{C}_{p}^{\prime}(x)=\sum_{a \in \mathcal{L}} \frac{\partial \hat{c}_{a}\left(f_{a}\right)}{\partial f_{a}} \delta_{a p},
$$

and $\mu_{w}$ is the Lagrange multiplier associated with constraint (4) for O/D pair $w$.

Observe that condition (24) may be rewritten so that there exists an ordering of paths for each O/D pair whereby all used paths (that is, those with positive flow) have equal and minimal marginal total costs and the unused paths (that is, those with zero flow) have higher (or equal) marginal total costs than those of the used paths. Hence, in the S-O problem, according to the optimality condition (24), it is the marginal of the total cost on each used path connecting an $\mathrm{O} / \mathrm{D}$ pair which is equalized and minimal.

\section{An Example}

We now return to the Braess network in Section 2.5, but we determine the S-O pattern. The system-optimized path flow pattern satisfying condition (24) is given by: $x_{p_{1}}=3, x_{p_{2}}=3$, and $x_{p_{3}}=0$, which corresponds to the link flow pattern: $f_{a}=f_{c}=3, f_{b}=f_{d}=3$, with $f_{e}=0$. The marginals of the total costs on the paths are:

$$
\hat{C}_{p_{1}}^{\prime}=\hat{C}_{p_{2}}^{\prime}=116, \quad \hat{C}_{p_{3}}^{\prime}=130
$$

Observe that the U-O flow pattern for this problem is distinct from the S-O problem. Indeed, in S-O networks, the Braess paradox can never occur.

Consider now user link cost functions that are of the general form (3), where the cost on a link may depend also on the flow on this as well as other flows on the network, that is,

$$
c_{a}=c_{a}(f), \quad \forall a \in \mathcal{L}
$$

The system-optimization problem in the case of nonseparable user link cost functions becomes:

$$
\text { Minimize } \sum_{a \in \mathcal{L}} \hat{c}_{a}(f),
$$

where $\hat{c}_{a}(f)=c_{a}(f) \times f_{a}, \forall a \in \mathcal{L}$, subject to constraints (1), (4), and the nonnegativity assumption on the path flows..

The system-optimality conditions remain as in (24) but where now the marginal of the total cost on a path becomes, in the more general case:

$$
\hat{C}_{p}^{\prime}=\sum_{a, b \in \mathcal{L}} \frac{\partial \hat{c}_{b}(f)}{\partial f_{a}} \delta_{a p}, \quad \forall p \in P .
$$


Of course, if the total link cost functions are strictly convex, then there is a unique S-O link flow pattern.

\section{Remark 2}

An interesting question is, under which user link cost functions (if any), is the S-O solution the same as the U-O solution? This question is relevant since in networks with such cost functions users would behave individually in a way that is also optimal from a societal perspective. The answer is as follows: on a general network, for user link cost functions given by:

$$
c_{a}\left(f_{a}\right)=t_{a}^{0} f_{a}^{\beta}, \quad \forall a \in \mathcal{L},
$$

the U-O solution coincides with the $\mathrm{S}-\mathrm{O}$ solution, for $t_{a}^{0}>0$, for all links $a \in \mathcal{L}$ and with $\beta$ being a nonnegative constant (see Dafermos and Sparrow (1969) and Nagurney and Qiang (2009a)). Of course, for networks with special structure, the class of functions can be broadened for this equivalence to hold. Note that in networks with cost functions given by (29) with $\beta=0$, which are uncongested networks, since the cost on a link does not depend on the flow on the link, the S-O and U-O patterns coincide.

In terms of network design, one may consider a network as being robust, in a sense, if the S-O flow pattern coincides with the U-O flow pattern. Networks that are designed with cost functions as in (29) would then be robust, in this sense. However, note that such networks do not have any fixed cost terms. Hence, it would be difficult to identify/construct networks, in practice, with such features.

\subsection{A Relative Total Cost Index for Assessing Network Robustness}

We now recall an index based on the relative total cost that assesses the robustness of a network based on the two behavioral solution concepts, namely, the total cost evaluated under the user-optimizing flow pattern, denoted by $T C_{U-O}$, and the system-optimizing flow pattern, denoted-by $T C_{S-O}$, respectively, in the case of fixed demands. The index was

proposed by Nagurney and Qiang (2009b). In particular, $T C_{U-O}$ denotes the total cost on the network as given by expression (27), where the vector $f$ is the solution to the user-optimizing (or transportation network equilibrium) condition (8). On the other hand, $T C_{S-O}$ is the total cost on the network as given also by expression (27), but evaluated at the flow pattern given by the solution to the S-O problem (24). The total cost is an appropriate measure since it represents the total cost to society associated with routing flows on networks. Furthermore, as links degrade and the practical capacity of links decreases the total cost is expected to increase and, hence, the relative total cost of a network reflects its robustness. Since we are 
considering robustness relative to changes in link capacities, the BPR functional form for link costs is relevant as are other link cost functions with explicit embedded capacity terms. We assume in this Section that the demands are fixed (and known).

The relative total cost index for a network $\mathcal{G}$ with the vector of fixed demands $d$, the vector of user link cost functions $c$, and the vector of link capacities $u$ is defined as the relative total cost increase under a given uniform capacity retention ratio $\gamma(\gamma \in(0,1])$ so that the new capacities are given by $\gamma u$. Let $c$ denote the vector of BPR user link cost functions (cf. (14)) and let $d$ denote the vector of $\mathrm{O} / \mathrm{D}$ demands.

\section{Definition 11: Relative Total Cost Index Under U-O Behavior}

The mathematical definition of the index under the user-optimizing flow pattern, denoted by $\mathcal{I}_{U-O}^{\gamma}$, is then:

$$
\mathcal{I}_{U-O}^{\gamma}=\mathcal{I}_{U-O}(G, c, d, \gamma, u)=\frac{T C_{U-O}^{\gamma}-T C_{U-O}}{T C_{U-O}} \times 100 \%,
$$

where $T C_{U-O}$ and $T C_{U-O}^{\gamma}$ are the total network costs evaluated under the user-optimizing flow pattern with the original capacities and the remaining capacities (i.e., ru), respectively.

\section{Definition 12: Relative Total Cost Index Under S-O Behavior}

The mathematical definition of the index under the system-optimizing flow pattern is:

$$
\mathcal{I}_{S-O}^{\gamma}=\mathcal{I}_{S-O}(G, c, d, \gamma, u)=\frac{T C_{S-O}^{\gamma}-T C_{S-O}}{T C_{S-O}} \times 100 \%
$$

where $T C_{S-O}$ and $T C_{S-O}^{\gamma}$ are the total network costs evaluated at the system-optimizing flow pattern with the original capacities and the remaining capacities (i.e., $\gamma u$ ), respectively.

From the above definition(s), a network, under a given capacity retention / deterioration ratio $\gamma$ (and either $\mathrm{S}-\mathrm{O}$ or $\mathrm{U}-\mathrm{O}$ behavior) is considered to be robust if the index $\mathcal{I}^{\gamma}$ is low. This means that the relative total cost does not change much and, hence, the transportation network may be viewed as being more robust than if the relative total cost is small.

\section{Remark 3}

We can also study the relative total cost improvement after capacity enhancement. In that case, since the relative total cost saving needs to be computed, we reverse the order of substraction in (30) and (31) with $\gamma \geq 1$. Furthermore, $\gamma$ is defined as the "Capacity Increment Ratio." Therefore, the larger the relative total cost index is, the more total cost savings can a capacity enhancement plan bring for a specific $\gamma$. 
The price of anarchy (cf. Roughgarden (2005), and the references therein), which is denoted by $\rho$, is defined as:

$$
\rho=\frac{T C_{U-O}}{T C_{S-O}}
$$

Observe that $\rho$ captures the relationship between total costs across distinct behavioral principles whereas the indices (30) and (31) are focused on the degradation of network performance within U-O or S-O behavior. Nevertheless, we have the following relationship between the ratio of the two indices and the price of anarchy, due to Nagurney and Qiang (2009a)

$$
\frac{I_{S-O}^{\gamma}}{I_{U-O}^{\gamma}}=\frac{\left[T C_{S-O}^{\gamma}-T C_{S-O}\right]}{\left[T C_{U-O}^{\gamma}-T C_{U-O}\right]} \times \rho .
$$

The term preceding the price of anarchy in (33) may be less than 1, greater than 1, or equal to 1 , depending upon the network and data.

As established in Roughgarden (2003), the price of anarchy is bounded by $O\left(\frac{\beta}{\log \beta}\right)$ when the cost function (sometimes also referred to in the computer science literature as a latency function) on each link is a polynominal function with nonnegative coefficients and degree at most $\beta$. This is the form, for example, of the BPR function. When $\beta=4$, then we have that the price of anarchy $\rho$ is bounded by $O\left(\frac{\beta}{\log \beta}\right)=6.6439$ for $\beta=4$.

\subsection{An Application to the Anaheim Network}

We now evaluate the relative total cost indices $I_{U-O}^{\gamma}$ and $I_{S-O}^{\gamma}$ for the Anaheim network to determine its robustness under alternative user behaviors. In Figure 7, we graph the ratio $I_{U-O}^{\gamma}$ to $I_{S-O}^{\gamma}$ for this network. We can see that the Anaheim network under the S-O solution is more robust in terms of the relative total cost increase when the capacity retention ratio $\gamma$ is above .3, whereas the $\mathrm{U}-\mathrm{O}$ solution leads to lower relative total cost increases; therefore, the network is more robust when $\gamma$ is below .3.

Figure 8, in turn, depicts the ratio of $I_{U-O}^{\gamma}$ to $I_{S-O}^{\gamma}$ for the Anaheim network when the capacity is enhanced (cf. Remark 3). When the capacity incremental ratio $\gamma$ is below 1.2, the U-O solution leads to a better relative total cost improvement in the Anaheim network, whereas the $\mathrm{S}-\mathrm{O}$ solution results in a better relative total cost saving when $\gamma$ is above 1.3. 


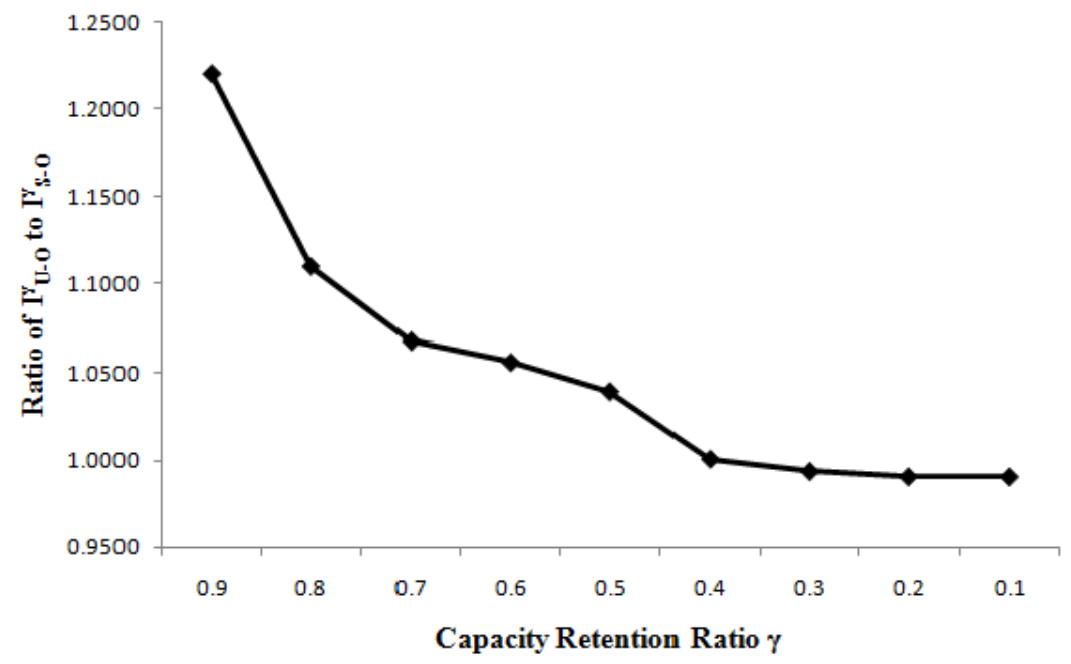

Figure 7: Ratio of $I_{U-O}^{\gamma}$ to $I_{S-O}^{\gamma}$ for the Anaheim Network Under Capacity Retention Ratio $\gamma$

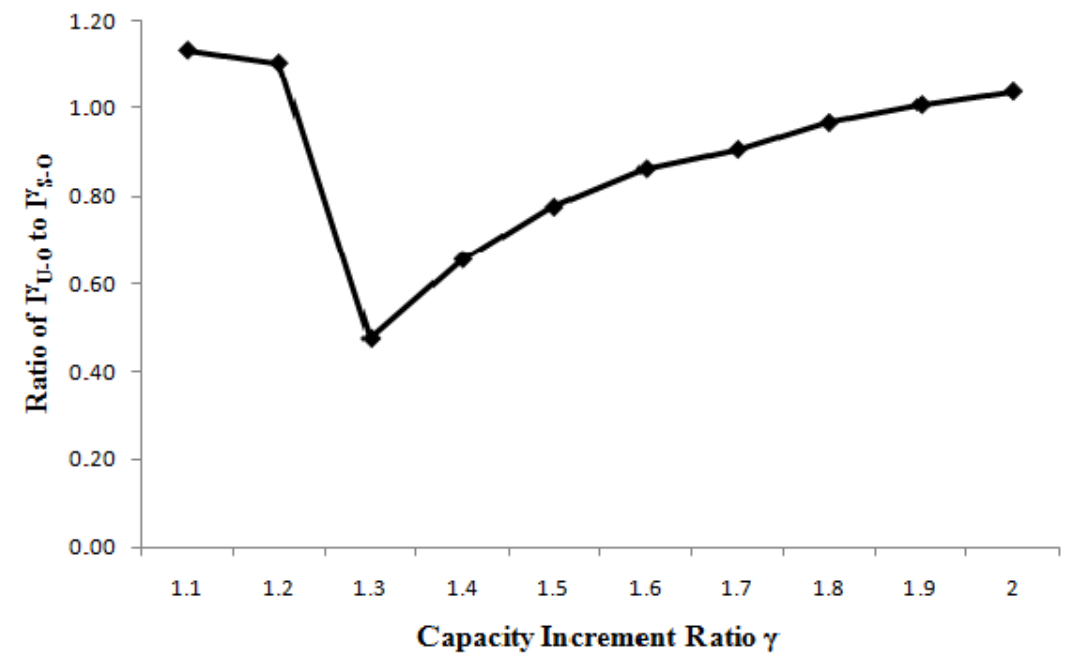

Figure 8: Ratio of $I_{U-O}^{\gamma}$ to $I_{S-O}^{\gamma}$ for the Anaheim Network Under Capacity Increment Ratio $\gamma$ 


\section{Network Integration and Associated Synergy}

In this Section, the use of a system-optimization perspective enables the modeling of the economic activities associated with a firm as a network and, hence, the evaluation of the strategic advantages, often referred to as synergy, due to mergers (or acquisitions), in a network format. However, unlike the classical system-optimization formulation, which was originally proposed in the context of transportation networks, and was recalled in Section 3 , here we explicitly consider capacities on the links of the networks. According to Soylu et al. (2006) more and more companies now realize the strategic importance of controlling the supply chain as a whole. Min and Zhou (2002) emphasized the need to analyze the synergy obtained through both interfunctional and interorganizational integration. Hakkinen et al. (2004) further described the integration of logistics after M\&As with a review of the literature and concluded that logistics issues have received insufficient attention (see also Herd, Saksena, and Steger (2005)).

We represent the underlying associated networks before and after the horizontal merger and demonstrate that the solution of all the associated system-optimization problems can be obtained by solving a variational inequality problem, with a structure that can be easily exploited for computational purposes. Notably, our framework incorporates manufacturing/production activities as well as distribution and storage activities both before and after the merger (or acquisition). Of course, whether a manufacturing plant or a distribution center is used before or after a merger depends on the optimal solution.

We also present a measure of strategic advantage, which allows one to evaluate the gains, if any, associated with the horizontal merger. The framework, originated by Nagurney (2009), is applicable to corporate as well as humanitarian operations settings.

\subsection{The Pre- and Post-Merger Supply Chain Network Models}

In this Section, we outline the supply chain network models before and after the horizontal merger. We consider two firms, denoted by Firm $A$ and Firm $B$, which are integrated after the merger. We assume that each firm produces the same homogeneous product because the focus here is on horizontal mergers in the same industry. The formalism that we use is that of system-optimization, by which each of the firms is assumed to own its manufacturing facilities/plants and distribution centers, and each firm seeks to determine the optimal production of the product at each of its manufacturing plants and the optimal quantities shipped to the distribution centers, where the product is stored and from which it is shipped to the retail outlets. Each firm seeks to minimize the total costs associated with 


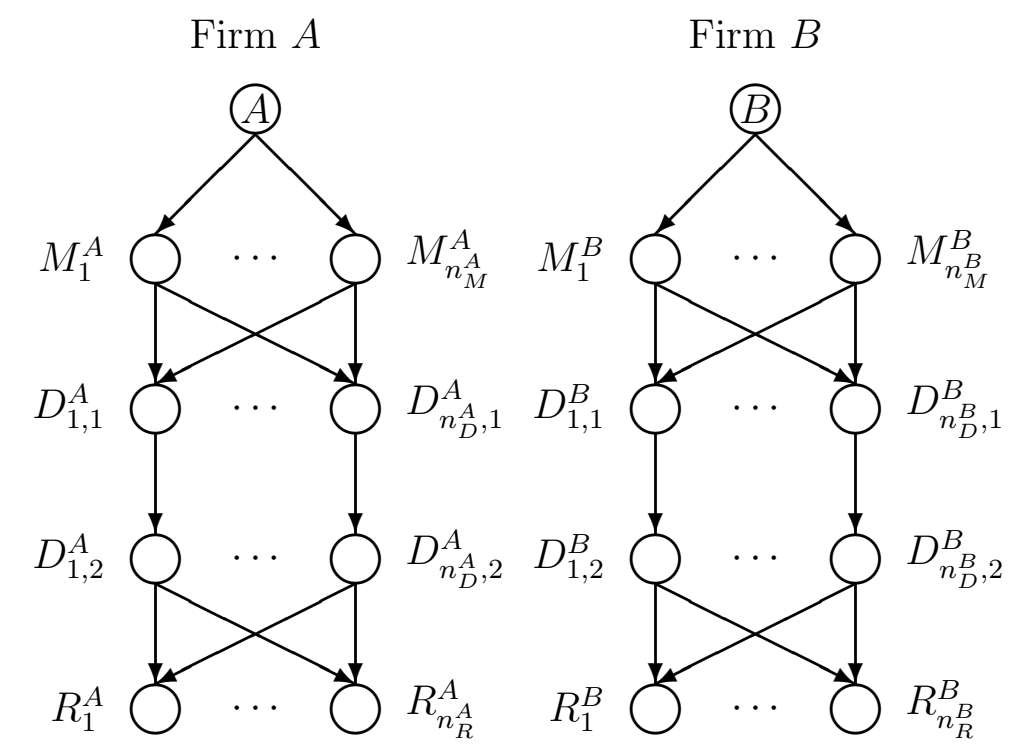

Figure 9: Case 0: Firms $A$ and $B$ Pre-Merger

the production, storage and distribution activities, subject to the demand being satisfied at the retail outlets. An appropriate time horizon for the merger is assumed.

In Section 4.1.1, we formulate the pre-merger system-optimization problem and in Section 4.1.2, we formulate the post-merger model.

\subsubsection{The Pre-Merger Network Model}

The optimization problem faced by Firm $A$ and Firm $B$ is as follows. Each firm is represented as a network of its economic activities, as depicted in Figure 9. Each firm $i ; i=A, B$, has $n_{M}^{i}$ manufacturing facilities/plants; $n_{D}^{i}$ distribution centers, and serves $n_{R}^{i}$ retail outlets. Let $\mathcal{G}_{i}=\left[\mathcal{N}_{i}, \mathcal{L}_{i}\right]$ for $i=A, B$ denote the graph consisting of nodes and directed links representing the economic activities associated with each firm $i$. Let $\mathcal{G}^{0}=\left[\mathcal{N}^{0}, \mathcal{L}^{0}\right] \equiv \cup_{i=A, B}\left[\mathcal{N}_{i}, \mathcal{L}_{i}\right]$. The links from the top-tiered nodes $i ; i=A, B$ in each network in Figure 9 are connected to the manufacturing nodes of the respective firm $i$, which are denoted, respectively, by: $M_{1}^{i}, \ldots, M_{n_{M}^{i}}^{i}$, and these links represent the manufacturing links.

The links from the manufacturing nodes, in turn, are connected to the distribution center nodes of each firm $i ; i=A, B$, which are denoted by $D_{1,1}^{i}, \ldots, D_{n_{D^{i}, 1}}^{i}$. These links correspond to the shipment links between the manufacturing plants and the distribution centers where the product is stored. The links joining nodes $D_{1,1}^{i}, \ldots, D_{n_{D}^{i}, 1}^{i}$ with nodes $D_{1,2}^{i}, \ldots, D_{n_{D}^{i}, 2}^{i}$ for $i=A, B$ correspond to the storage links. Finally, there are shipment links joining the nodes 
$D_{1,2}^{i}, \ldots, D_{n_{D}^{i}, 2}^{i}$ for $i=A, B$ with the retail outlet nodes: $R_{1}^{i}, \ldots, R_{n_{R}^{i}}^{i}$ for each firm $i=A, B$. Note that each firm $i$ has its individual retail outlets where it sells the product, as depicted in Figure 9.

Assume that associated with each link (cf. Figure 9) of the network corresponding to each firm $i ; i=A, B$ is a total cost. We denote, without any loss in generality, the links by $a, b$, etc., and the total cost on a link $a$ by $\hat{c}_{a}$. The demands for the product are assumed as given and are associated with each firm and retailer pair. Let $d_{R_{k}^{i}}$ denote the demand for the product at retailer $R_{k}^{i}$ associated with firm $i ; i=A, B ; k=1, \ldots, n_{R}^{i}$. Let $x_{p}$ denote the nonnegative flow of the product on path $p$ joining (origin) node $i$ with a (destination) retailer node of firm $i ; i=A, B$. Then the following conservation of flow equations must hold for each firm $i$

$$
d_{R_{k}^{i}}=\sum_{p \in P_{R_{k}^{i}}^{0}} x_{p}, \quad i=A, B ; k=1, \ldots, n_{R}^{i},
$$

where $P_{R_{k}^{i}}^{0}$ denotes the set of paths connecting (origin) node $i$ with (destination) retail node $R_{k}^{i}$. In other words, the demand for the product at each retail outlet associated with each firm must be satisfied by the sum of the product flows from the firm to that retail outlet. Note that a path consists of a sequence of links representing the economic activities of manufacturing/production, distribution, storage, and final shipment to the retailer.

In addition, let $f_{a}$ denote the flow of the product on link $a$. Hence the following conservation of flow equations must also hold

$$
f_{a}=\sum_{p \in P^{0}} x_{p} \delta_{a p}, \quad \forall a \in \mathcal{L}^{0}
$$

where $\delta_{a p}=1$ if link $a$ is contained in path $p$ and $\delta_{a p}=0$, otherwise, that is, the (product) flow on a link is equal to the sum of the (product) path flows on paths that contain that link. $P^{0}$ denotes the set of all paths in Figure 9, that is, $P^{0}=\cup_{i=A, B ; k=1, \ldots, n_{R}^{i}} P_{R_{k}^{i}}^{0}$. Because here we consider the two firms before any merger the paths associated with a given firm have no links in common with paths of the other firm. This changes when the horizontal merger occurs, in which case the set of paths and the number of paths also change, as does the set of links and the number of links, as we demonstrate below.

Of course, the path flows must be nonnegative, that is

$$
x_{p} \geq 0, \quad \forall p \in P^{0} .
$$

The total cost on a link, be it a manufacturing/production link, a shipment link, or a storage link is assumed to be a function of the flow of the product on the link. Hence we 
have

$$
\hat{c}_{a}=\hat{c}_{a}\left(f_{a}\right), \quad \forall a \in \mathcal{L}^{0}
$$

The total cost on each link is assumed to be convex, continuously differentiable, and with a bounded second order partial derivative. The same is assumed to hold for all links that are added post the merger. Such conditions will guarantee convergence of the proposed algorithm.

There are positive capacities on the links with the capacity on link $a$ denoted by $u_{a}$, for all links $a \in \mathcal{L}^{0}$. This is very reasonable because the manufacturing plants, the shipment links, and the distribution centers, which serve also as the storage facilities, can be expected to have capacities, in practice.

The total cost associated with the economic activities of both firms before the merger is minimized when the following system-optimization problem is solved

$$
\text { Minimize } \sum_{a \in \mathcal{L}^{0}} \hat{c}_{a}\left(f_{a}\right)
$$

subject to: constraints $(34)-(36)$ and

$$
f_{a} \leq u_{a}, \quad \forall a \in \mathcal{L}^{0}
$$

The solution of the above optimization problem will minimize the total costs associated with each firm individually and both firms together because they are, before the merger, independent and share no manufacturing facilities or distribution facilities or retail outlets. Observe that this problem is a system-optimization problem (see also Section 3) but in capacitated form. Under the above imposed assumptions, the optimization problem is a convex optimization problem. If we further assume that the feasible set underlying the problem represented by the constraints (34) through (36) and (39) is non-empty, then it follows from the standard theory of nonlinear programming (cf. Bazaraa, Sherali, and Shetty (1993)) that the optimal solution, denoted by $f^{*} \equiv\left\{f_{a}^{*}\right\}, a \in \mathcal{L}^{0}$, exists. If the total cost functions (37) are strictly convex, then this link flow solution is unique. Let $\mathcal{K}^{0}$ denote the feasible set: $\mathcal{K}^{0} \equiv\{f \mid \exists x \geq 0$, and (34) $-(36)$ hold $\}$.

Associated with constraint (39) for each link $a$ is the Lagrange multiplier $\beta_{a}$, with the optimal Lagrange multiplier denoted by $\beta_{a}^{*}$. This term may be interpreted as the price or value of an additional unit of capacity on link $a$ for each $a \in \mathcal{L}^{0}$.

The variational inequality formulation of the problem is given below. 


\section{Theorem 5: Variational Inequality Formulation of the Network Pre-Integration Problem}

The vector of link flows $f^{*} \in \mathcal{K}^{0}$ is an optimal solution to problem (38), subject to (34) through (36) and (39), if and only if it satisfies the following variational inequality problem with the vector of optimal nonnegative Lagrange multipliers $\beta^{*}$

$\sum_{a \in \mathcal{L}^{0}}\left[\frac{\partial \hat{c}_{a}\left(f_{a}^{*}\right)}{\partial f_{a}}+\beta_{a}^{*}\right] \times\left[f_{a}-f_{a}^{*}\right]+\sum_{a \in \mathcal{L}^{0}}\left[u_{a}-f_{a}^{*}\right] \times\left[\beta_{a}-\beta_{a}^{*}\right] \geq 0, \quad \forall f \in \mathcal{K}^{0}, \quad \forall \beta_{a} \geq 0, \forall a \in \mathcal{L}^{0}$.

This variational inequality can be easily solved using the modified projection method of Korpelevich (1977). The elegance of this computational procedure in the context of variational inequality (40) lies in that it allows one to utilize algorithms for the solution of the uncapacitated system-optimization problem (for which numerous algorithms exist in the transportation science literature) with a straightforward update procedure at each iteration to obtain the Lagrange multipliers. The variational inequality governing the supply chain network post-merger will also be of the form (40) and thus amenable to solution by the modified projection method. The modified projection method is guaranteed to converge to a solution of a variational inequality problem, provided that the function that enters the variational inequality problem is monotone and Lipschitz continuous and that a solution exists.

Once we have solved problem (40) we have the solution $f^{*}$ which minimizes the total cost (38) in the supply chain networks associated with the two firms. We denote this total cost given by $\sum_{a \in \mathcal{L}^{0}} \hat{c}_{a}\left(f_{a}^{*}\right)$ as $T C^{0}$, and we use this total cost value as a baseline from which to compute the strategic advantage or synergy, discussed in Section 4.2, associated with horizontal merger that we describe next.

\subsubsection{The Post-Merger Network Model}

In this Section, the firms merge and the retailers can obtain the product produced at any of the manufacturing facilities and distributed by any of the distribution centers, as in Figure 10. We now formulate the merger in which Firms $A$ and $B$ merge and the retailers can obtain the product from any manufacturer and shipped from any distribution center. Figure 10 depicts the network topology associated with this type of horizontal merger. We refer to the network underlying this merger as $\mathcal{G}^{1}=\left[\mathcal{N}^{1}, \mathcal{L}^{1}\right]$.

Let $x_{p}$, without loss of generality, denote the flow of the product on path $p$ joining (origin) 


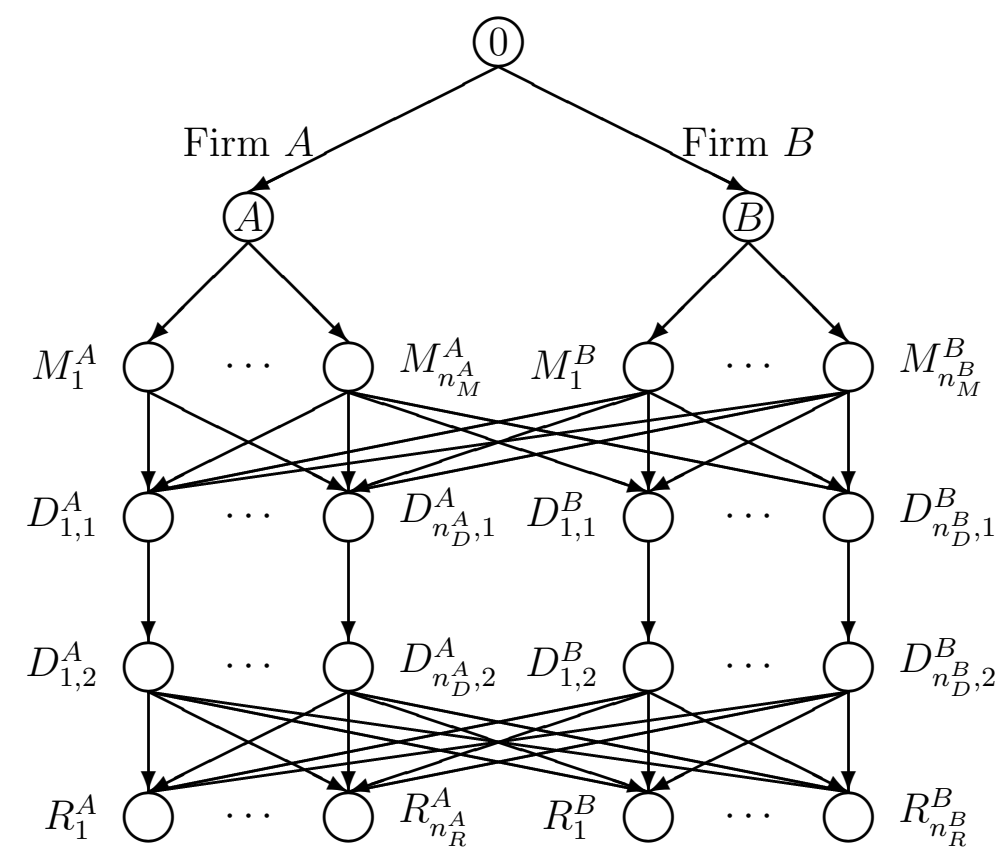

Figure 10: Post-Merger Network

node 0 with a (destination) retailer node as in Figure 10. Then the following conservation of flow equations must hold

$$
d_{R_{k}^{i}}=\sum_{p \in P_{R_{k}^{i}}^{1}} x_{p}, \quad i=A, B ; k=1, \ldots, n_{R}^{i},
$$

where $P_{R_{k}^{i}}^{1}$ denotes the set of paths connecting node 0 with retail node $R_{k}^{i}$ in Figure 10 . The set of paths $P^{1} \equiv \cup_{i=A, B ; k=1, \ldots, n_{R}^{i}} P_{R_{k}^{i}}^{1}$.

In addition, as before, let $f_{a}$ denote the flow of the product on link $a$. Hence the following conservation of flow equations must now be satisfied

$$
f_{a}=\sum_{p \in P^{1}} x_{p} \delta_{a p}, \quad \forall a \in \mathcal{L}^{1} .
$$

These path flows must also be nonnegative since they represent products, that is

$$
x_{p} \geq 0, \quad \forall p \in P^{1} .
$$

The optimization problem associated with this horizontal merger, which minimizes the total cost of the network in Figure 10, is

$$
\text { Minimize } \sum_{a \in \mathcal{L}^{1}} \hat{c}_{a}\left(f_{a}\right)
$$


subject to: constraints $(41)-(43)$ and

$$
f_{a} \leq u_{a}, \quad \forall a \in \mathcal{L}^{1}
$$

The solution to this problem can also be obtained as a solution to a variational inequality problem akin to (40), where $a \in \mathcal{L}^{1}$, and the variable and solution vectors are as defined before but with expanded dimensions. Finally, instead of $\mathcal{K}^{0}$ we now have $\mathcal{K}^{1} \equiv\{f \mid \exists x \geq$ 0 , and $(41)-(43)$ hold $\}$. One can also apply the modified projection problem to compute the solution to the variational inequality problem governing the merger. The total cost $T C^{1}$, which is the value of the objective function (44) evaluated at its optimal solution $f^{*}$, is equal to $\sum_{a \in \mathcal{L}^{1}} \hat{c}_{a}\left(f_{a}^{*}\right)$. In the next Section, we discuss how we use the total costs: $T C^{0}$ and $T C^{1}$, to determine the strategic advantage (or synergy) associated with the horizontal merger.

\subsection{Measuring the Strategic Advantage Associated with Horizontal Mergers}

We now provide a measure for quantifying the strategic advantage or synergy associated with the merger. The synergy measure that we utilize to capture the gains, if any, associated with a merger is denoted by $\mathcal{S}$, and is given by:

$$
\mathcal{S}=\left[\frac{T C^{0}-T C^{1}}{T C^{0}}\right] \times 100 \% .
$$

\subsection{A Numerical Example}

In this Section, we present a numerical example for which we compute the strategic advantage measure as in (46). We consider Firm $A$ and Firm $B$, each of which has two manufacturing plants: $M_{1}^{i}$ and $M_{2}^{i} ; i=A, B$. In addition, each firm has a single distribution center to which the product is shipped from the manufacturing plants and stored. Finally, once stored, the product is shipped to the two retailers associated with each firm and denoted by $R_{1}^{i}$ and $R_{2}^{i}$ for $i=A, B$. A graphical depiction of the supply chain networks associated with the two firms pre-merger is given in Figure 11. Figure 12 depicts the merger of these two firms.

We used the modified projection method, embedded with the equilibration algorithm, to compute the solutions to the problems (see also Nagurney (2009) for additional examples and details). We implemented the algorithm in FORTRAN and used a Unix system at the University of Massachusetts for the computations. 


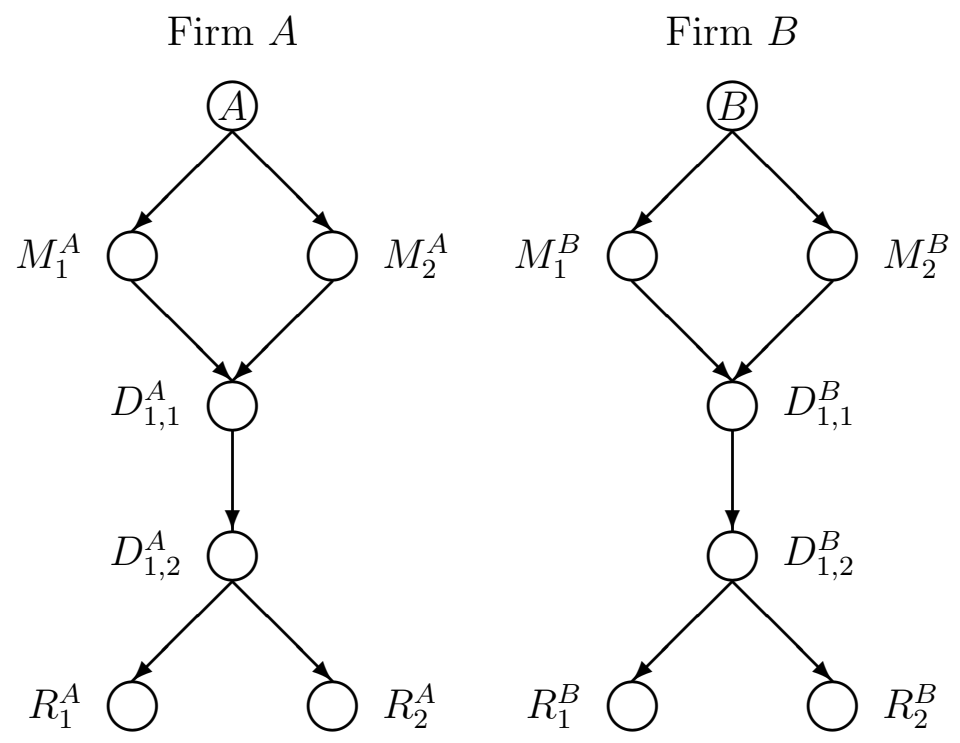

Figure 11: Network Topology for the Numerical Example Pre-Merger

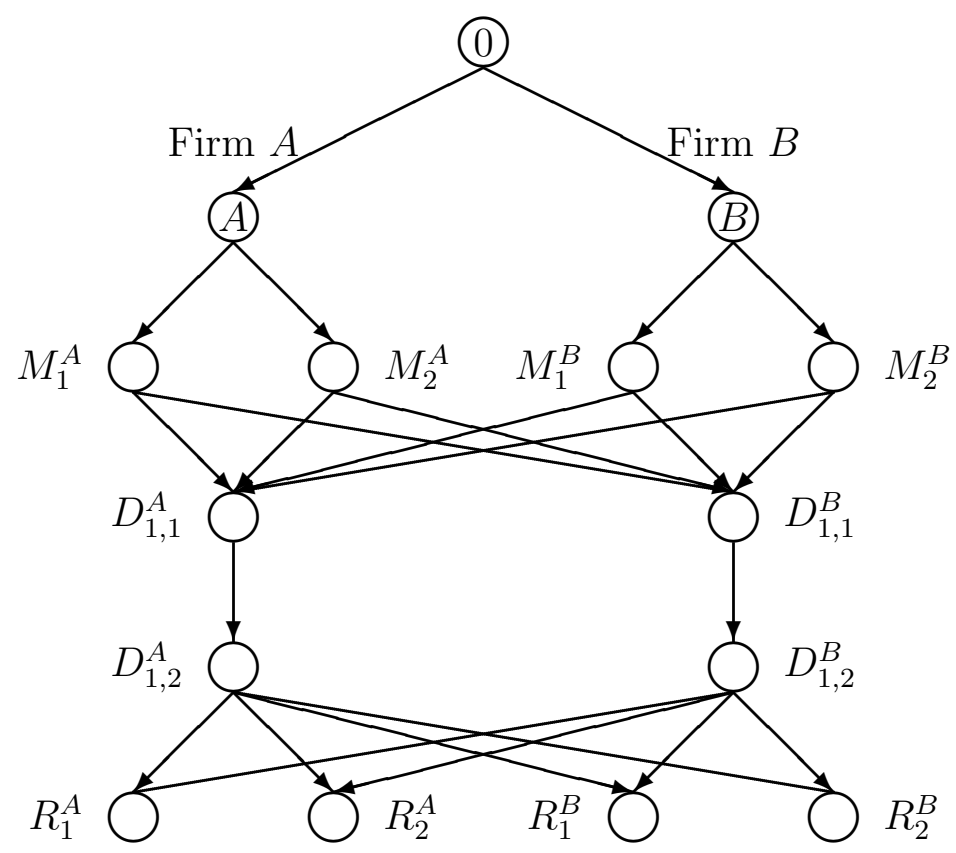

Figure 12: Network Topology for the Numerical Example Post-Merger 
Table 5: Definition of Links and Associated Total Cost Functions for the Merger Example

\begin{tabular}{|c|c|c|c|}
\hline $\begin{array}{c}\text { Link } \\
a\end{array}$ & $\begin{array}{c}\text { From } \\
\text { Node }\end{array}$ & $\begin{array}{c}\text { To } \\
\text { Node }\end{array}$ & $\begin{array}{c}\text { Total Cost } \\
\text { Function }\end{array}$ \\
\hline 1 & $A$ & $M_{1}^{A}$ & $.5 f_{1}^{2}+f_{1}$ \\
\hline 2 & $A$ & $M_{2}^{A}$ & $.5 f_{2}^{2}+f_{2}$ \\
\hline 3 & $M_{1}^{A}$ & $D_{1,1}^{A}$ & $f_{3}^{2}+2 f_{3}$ \\
\hline 4 & $M_{2}^{A}$ & $D_{1,1}^{A}$ & $f_{4}^{2}+2 f_{4}$ \\
\hline 5 & $D_{1,1}^{A}$ & $D_{1,2}^{A}$ & $.5 f_{5}^{2}+f_{5}$ \\
\hline 6 & $D_{1,2}^{A}$ & $R_{1}^{A}$ & $f_{6}^{2}+2 f_{6}$ \\
\hline 7 & $D_{1,2}^{A}$ & $R_{2}^{A}$ & $f_{7}^{2}+2 f_{7}$ \\
\hline 8 & $B$ & $M_{1}^{B}$ & $f_{8}^{2}+2 f_{8}$ \\
\hline 9 & $B$ & $M_{2}^{B}$ & $f_{9}^{2}+2 f_{9}$ \\
\hline 10 & $M_{1}^{B}$ & $D_{1,1}^{B}$ & $f_{10}^{2}+2 f_{10}$ \\
\hline 11 & $M_{2}^{B}$ & $D_{1,1}^{B}$ & $f_{11}^{2}+2 f_{11}$ \\
\hline 12 & $D_{1,1}^{B}$ & $D_{1,2}^{B}$ & $.5 f_{12}^{2}+f_{12}$ \\
\hline 13 & $D_{1,2}^{B}$ & $R_{1}^{B}$ & $f_{13}^{2}+2 f_{13}$ \\
\hline 14 & $D_{1,2}^{B}$ & $R_{2}^{B}$ & $f_{14}^{2}+2 f_{14}$ \\
\hline 15 & $M_{1}^{A}$ & $D_{1,1}^{B}$ & $f_{15}^{2}+2 f_{15}$ \\
\hline 16 & $M_{2}^{A}$ & $D_{1,1}^{B}$ & $f_{16}^{2}+2 f_{16}$ \\
\hline 17 & $M_{1}^{B}$ & $D_{1,1}^{A}$ & $f_{17}^{2}+2 f_{17}$ \\
\hline 18 & $M_{2}^{B}$ & $D_{1,1}^{A}$ & $f_{18}^{2}+2 f_{18}$ \\
\hline 19 & $D_{1,2}^{A}$ & $R_{1}^{B}$ & $f_{19}^{2}+2 f_{19}$ \\
\hline 20 & $D_{1,2}^{A}$ & $R_{2}^{B}$ & $f_{20}^{2}+2 f_{20}$ \\
\hline 21 & $D_{1,2}^{B}$ & $R_{1}^{A}$ & $f_{21}^{2}+2 f_{21}$ \\
\hline 22 & $D_{1,2}^{B}$ & $R_{2}^{A}$ & $f_{2}^{2}+2 f_{22}$ \\
\hline 23 & 0 & $A$ & .00 \\
\hline 24 & 0 & $B$ & .00 \\
\hline
\end{tabular}

In Table 5, we define the links on the two networks, and the total link cost functions associated with the various supply chain activities of manufacturing, shipping/distribution, and storage. The merger links (emanating from node 0) are assumed to have associated total costs of zero. The capacities on all the links were set to 15 . The demands at the retailers were $d_{R_{1}^{A}}=10, d_{R_{2}^{A}}=5$, and $d_{R_{1}^{B}}=5, d_{R_{2}^{B}}=5$.

In Table 6 we report the total costs and the synergy associated with the merger, which is equal to $24.1 \%$, a not insignificant percent.

The synergy measure $\mathcal{S}$ in (46) has been generalized to include environmental cost in terms of a generalized cost associated with multicriteria decision-making associated with network integration by Nagurney and Woolley (2010). Nagurney, Woolley, and Qiang (2010), 
Table 6: Total Costs and Synergy of the Merger Example

\begin{tabular}{|c|c|}
\hline$T C^{0}$ & 766.25 \\
\hline$T C^{1}$ & 581.30 \\
\hline $\mathcal{S}$ & $24.1 \%$ \\
\hline
\end{tabular}

in turn, developed a multiproduct network model which allows for the determination of synergy associated with network integration in that more general setting which is applicable not only to corporate mergers and acquisitions but also to humanitarian operations. For example, Nagurney and Qiang (2009a) have emphasized the relevance of such network models for the quantification of synergy associated with the collaboration of teams or partners in humanitarian logistics operations. In the context of humanitarian logistics, one would consider, in terms of the above framework, the specific humanitarian organizations (rather than firms) and their integration for a particular humanitarian disaster relief operation. Rather than manufacturing links, one would have supply links with associated total costs of procurement. Since the public who donates funds expects an appropriate utilization of the financial resources, the framework described here could quantify the total costs and the synergy obtained through cooperation. Of course, one could also conduct sensitivity analysis to evaluate a spectrum of possible demands as well as a range of link capacities to make sure that the demand can be satisfied. Moreover, one might incorporate also ideas from multicriteria decision-making, since it can be expected that, during times of disasters and associated uncertainty, risk is a very important issue in disaster relief operations and the total cost functions could be generalized costs to capture risk.

Also, it is worth noting that in this Section we have focused on network integration and synergy in a system-optimization context. Nagurney (2008) developed a network oligopoly model in which firms compete and consumers at demand markets take the prices of the product (which are elastic) into consideration in selecting the product. She showed that by exploiting fully the underlying network structure of firms' economic activities that one could gain insights into what is known as the merger paradox (Creane and Davidson (2004)).

Finally, we emphasize that the framework and concepts on network integration discussed here, coupled with the work of Nagurney (2008), may be applied to the integration of firms in a variety of network industries from telecommunications, transportation (railways and airlines), financial services, and energy, including electric power. 


\section{Recent Research on Network Design and Suggestions for Future Research}

This paper presented an overview of some of the recent developments in the assessment of network vulnerability and robustness through appropriate tools that assist in the quantification of network efficiency / performance and the identification of the importance of network components, such as nodes and links. We demonstrated how rigorously constructed and well-defined network measures can capture not only the network topology underlying a particular critical system, but also the underlying behavior of decision-makers, the resulting flows, and induced costs in the reality of demands for resources, whether fixed or elastic (price-dependent). In addition, we reviewed how to determine the synergy associated with network integration, with a focus on supply chains, as may occur not only in corporate applications, such as in mergers and acquisitions, but also in humanitarian ones, as in the case of the creation of teams and partnerships for humanitarian logistics.

We illustrated the concepts and tools in this paper, which are based on numerous publications, including the recent book by Nagurney and Qiang (2009a), through a spectrum of applications and numerical examples. Due to space limitations, we presented the highlights of the research in a careful, selective manner. Since the number of disasters is growing globally, it is imperative to have transparent, well-understood, and appropriate tools for the determination of network vulnerability and robustness, since networks from transportation, telecommunications, manufacturing and logistical ones, to financial and electric power ones, including, the underdevelopment, smart grid (see ISO New England (2009)), provide the ties that bind our economies and societies together. Local disruptions can have global impacts. Only when network components are identified as to their importance and rankings can decision-makers and policy analysts as well as planners and engineers understand in an objective way which components should be maintained and protected the most.

The present research agenda that is called for is interdisciplinary and broad. Of particular relevance, we believe, is the development of mathematical models and algorithms for network design problems, which can handle some of the major issues of today. For example, Nagurney, $\mathrm{Yu}$, and Qiang (2009) developed an integrated framework for the design of supply chain networks for critical products such as vaccines, medicines, food, etc., which may be used in preparation (and response) to pandemics, disasters, attacks, etc. The model utilizes cost

minimization within a system-optimization perspective as the primary objective and captures rigorously the uncertainty associated with the demand for critical products at the various demand points. In addition, the supply chain network design model allows for the investment of enhanced link capacities associated with such supply chain activities as manufacturing, storage, and distribution. Moreover, it allows for nonnegative initial capacities on the supply 
chain activities that the organization controls. The organization contracts the outsource product volumes at a fixed price. Finally, the model allows for the investigation of whether the product should be outsourced or produced in-house.

The methodology that we utilize for the formulation and solution of the supply chain network design model for critical needs is that of the theory of variational inequalities. The framework can be applied in situations in which the goal is to produce and deliver a critical product at minimal cost so as to satisfy the demand at various demand points, as closely as possible, given associated penalties for under-supply (and, if also relevant, for over-supply, which we expect to be lower than the former). Such a network design model is relevant to vaccine production as well as to emergency preparedness and humanitarian logistics. We believe that it is a step in the direction of designing networks with robust features in mind.

Network design, however, in the case of competing, profit-maximizing firms, as in oligopolies, with examples being airlines, oil companies, wireless companies, among others, would need to take advantage of game theoretic constructs. Nagurney (2010) developed an oligopolistic network design model and formulated the governing Nash equilibrium conditions as a variational inequality problem, whose solution yields both link capacities as well as link product flows.

Finally, we believe that the ideas in this paper, coupled with some of our recent research in network design and sustainability (see also Nagurney and Nagurney (2009)) have direct relevance to energy networks of the 21st century, such as the smart grid, which will involve decentralized decision-making, the use of renewable sources of energy, smart appliances, advanced information technology, sensors, and dynamic pricing, to transform electric power provision in modern societies. As the smart grid evolves, attention must be placed on ensuring its robustness.

\section{Acknowledgments}

The first author thanks Professor Celso Ribeiro, the Editor of the International Transactions in Operational Research, for the opportunity to write this paper, which is based on her tutorial prepared for the ALIO-INFORMS Joint International Meeting in Buenos Aires, June 6-9, 2010, and, which was inspired by the book, Fragile networks: identifying vulnerabilities and synergies in an uncertain world, by Nagurney and Qiang (2009a).

The authors acknowledge the helpful comments and suggestions of both the Editor and an anonymous reviewer on an earlier version of this paper. 
The authors thank Min Yu for her careful reading of the paper and for her helpful suggestions.

\section{References}

Ahuja, R. K., Magnanti, T. L., and Orlin, J. B. (1993), Network flows: theory, algorithms, and applications, Prentice-Hall, Upper Saddle River, New Jersey.

Amaral, L. A. N., Scala, A., Barthélèmy, M., Stanley, H. E., 2000. Classes of small-world networks. Proceedings of the National Academy of Sciences USA 97, 11149-11152.

American Society of Civil Engineers, 2005. Report card for America's infrastructure. http://www.asce.org/reportcard/2005/index.cfm Accessed on November 15, 2007.

Balcik, B., Beamon, B., 2008. Facility location in humanitarian relief. International Journal of Logistics: Research and Applications 11, 101-121.

Barabási, A. L., 2003. Linked: how everything is connected to everything else and what it means. Plume, New York.

Bar-Gera, H., 2002. Origin-based algorithm for the traffic assignment problem. Transportation Science 36, 398-417.

Bar-Gera, H., 2008. Transportation network test problems.

http://www.bgu.ac.il/ bargera/tntp/ Accessed on November 15, 2008.

Barrat, A., Barthélémy, M., Pastor-Satorras, R., Vespignani, A., 2004. The architecture of complex weighted networks. Proceedings of the National Academy of Sciences USA 101, 3747-3752.

Barrat, A., Barthélèmy, M., Vespignani, A., 2005. The effects of spatial constraints on the evolution of weighted complex networks. Journal of Statistical Mechanics: Theory and Experiment, Article no. P05003.

Bazaraa, M. S., Sherali, H. D., Shetty, C. M., 1993. Nonlinear programming: theory and algorithms. John Wiley \& Sons, New York.

Beckmann, M. J., McGuire, C. B., Winsten, C. B., 1956. Studies in the economics of transportation. Yale University Press, New Haven, Connecticut.

Bertsekas, D. P., Gallager, R. G., 1987. Data networks. Prentice-Hall, Englewood Cliffs, 
New Jersey.

Bonacich, P., 1972. Factoring and weighting approaches to status scores and clique identification. Journal of Mathematical Sociology 2, 13-20.

Boyce, D. E., Mahmassani, H. S., Nagurney, A., 2005. A retrospective on Beckmann, McGuire, and Winsten's studies in the economics of transportation. Papers in Regional Science 84, 85-103.

Braess, D., 1968. Uber ein paradoxon aus der verkehrsplanung. Unternehmensforschung 12, 258-268.

Braess, D., Nagurney, A., Wakolbinger, T., 2005. On a paradox of traffic planning, translation of the 1968 article by Braess. Transportation Science 39, 446-450.

Braine, T., 2006. Was 2005 the year of natural disasters? Bulletin of the World Health Organization 84, 1-80.

Bureau of Public Roads, 1964. Traffic assignment manual. U.S. Department of Commerce, Urban Planning Division, Washington, DC.

Callaway, D. S., Newman, M. E. J., Strogatz, S. H., Watts, D. J., 2000. Network robustness and fragility: percolation on random graphs. Physical Review Letters 85, 5468-5471.

Canadian Competition Bureau, 2006. Competition bureau concludes examination into gasoline price spike following hurricane Katrina.

http://www.competitionbureau.gc.ca Accessed on January 4, 2009.

Chari, V. V., Christiano, L. J., Kehoe, P. J., 2008. Facts and myths about the financial crisis of 2008. Working paper 666, The Federal Reserve Bank of Minneapolis, Minneapolis, Minnesota.

Chassin, D. P., Posse, C., 2005. Evaluating North American electric grid reliability using the Barabási-Albert network model. Physica A 355, 667-677.

Creane, A., Davidson, C., 2004. Multidivisional firms, internal competition and the merger paradox. Canadian Journal of Economics 37, 951-977.

Dafermos, S. C., 1971. An extended traffic assignment problem with applications to two-way traffic. Transportation Science 5, 366-389. 
Dafermos, S., 1980. Traffic equilibrium and variational inequalities. Transportation Science $14,42-54$.

Dafermos, S., 1982. The general multimodal network equilibrium problem with elastic demand. Networks 12, 57-72.

Dafermos, S. C., Sparrow, F. T., 1969. The traffic assignment problem for a general network. Journal of Research of the National Bureau of Standards 73B, 91-118.

Dall'Asta, L., Barrat, A., Barthélemy, M., Vespignani, A., 2006. Vulnerability of weighted networks. Journal of Statistical Mechanics, Article no. P04006.

Daniele, P., 2006. Dynamic networks and evolutionary variational inequalities. Edward Elgar Publishing, Cheltenham, England.

Dantzig, G. B., 1951. Application of the simplex method to the transportation problem. In Activity Analysis of Production and Allocation, T. C. Koopmans, editor, John Wiley \& Sons, New York, pp. 359-373.

Davidson, K. B., 1966. The theoretical basis of a flow-travel time relationship for use in transportation planning. Australian Road Research 8, 32-35.

Emergency Events Database, 2008. Center for Research on the Epidemiology of Disasters, Catholic University of Louvain, Belgium. http://www.emdat.be/ Accessed on December 15, 2008.

Federal Emergency Management Agency, 1992. Federal response plan. FEMA Publication 229.

Ford, L. R., Fulkerson, D. R., 1962. Flows in networks. Princeton University Press, Princeton, New Jersey.

Freeman, L. C., 1979. Centrality in social networks: Conceptual clarification. Social Networks 1, 215-239.

Freeman, L. C., Borgatti, S. P., White, D. R., 1991. Centrality in valued graphs: A measure of betweenness based on network flow. Social Networks 13, 141-154.

Hakkinen, L., Norrman, A., Hilmola, O.-P., Ojala, L., 2004. Logistics integration in horizontal mergers and acquisitions. The International Journal of Logistics Management 15, 27-42. 
Herd, T., Saksena, A. K., Steger, T. W., 2005. Delivering merger synergy: A supply chain perspective on achieving high performance. Outlook - Point of View, Accenture, May.

Hitchcock, F. L., 1941. The distribution of a product from several sources to numerous facilities. Journal of Mathematical Physics 20, 224-230.

Holmgren, A. J., 2007. A framework for vulnerability assessment of electric power systems. In Reliability and vulnerability in critical infrastructure: a quantitative geographic perspective. Murray, A., Grubesic, T., Editors, Springer, New York, pp. 31-55.

International Strategy for Disaster Reduction, 2006. Press Release. January 30, 2006. http://www.unisdr.org/eng/media-room/press-release/2006/ PR-2006-02-Disasters -increase-18-per-cent-2005-but-death-rates-drop.pdf Accessed on January 5, 2009.

Institute of Electrical and Electronics Engineers, 1990. IEEE standard computer dictionary: a compilation of IEEE standard computer glossaries. New York.

ISO New England, 2009. Overview of the smart grid. February 17, Holyoke, Massachusetts.

Jenelius, E., Petersen, T., Mattsson, L. G., 2006. Road network vulnerability: Identifying important links and exposed regions. Transportation Research A 20, 537-560.

Kantorovich, L. V., 1939. Mathematical methods in the organization and planning of production. Publication house of the Leningrad University; Translated in Management Science 6, 1960, 366-422.

König, D., 1936. Theorie der endlichen und unendlichen graphen. Teubner, Leipzig, Germany.

Koopmans, T. C., 1947. Optimum utilization of the transportation system. Proceedings of the International Statistical Conference, Washington DC; Also in Econometrica 17, 1949, 136-145.

Korilis, Y. A., Lazar, A. A., Orda, A., 1999. Avoiding the Braess paradox in non-cooperative networks. Journal of Applied Probability 36, 211-222.

Korpelevich, G. M., 1977. The extragradient method for finding saddle points and other problems. Matekon 13, 35-49.

Langabeer, J., 2003. An investigation of post-merger supply chain performance. Journal of Academy of Business and Economics 2, 14-25. 
Langabeer, J., Seifert, D., 2003. Supply chain integration: The key to merger success (synergy). Supply Chain Management Review 7, 58-64.

Latora, V., Marchiori, M., 2001. Efficient behavior of small-world networks. Physical Review Letters 87, Article no. 198701.

Latora, V., Marchiori, M., 2002. Is the Boston subway a small-world network? Physica A 314, 109-113.

Latora, V., Marchiori, M., 2004. How the science of complex networks can help developing strategies against terrorism. Chaos, Solitons and Fractals 20, 69-75.

Latour, A., 2001. Trial by fire: A blaze in Albuquerque sets off major crisis for cell-phone giants. Wall Street Journal, January 29.

Leventhal, T., Nemhauser, G., Trotter, L., 1973. A column generation algorithm for optimal traffic assignment. Transportation Science 7, 168-176.

Liu, Z., Nagurney, A., 2007. Financial networks with intermediation and transportation network equilibria: A supernetwork equivalence and computational management reinterpretation of the equilibrium conditions with computations. Computational Management Science 4, 243-281.

Liu, Z., Nagurney, A., 2009. An integrated electric power supply chain and fuel market network framework: theoretical modeling with empirical analysis for New England. Naval Research Logistics 56, 600-624.

Marks, M. L., Mirvis, P. H., 2001. Making mergers and acquisitions work: Strategic and psychological preparation. Academy of Management Executive 15, 80-92.

Min, H., Zhou, G. 2002. Supply chain modeling: Past, present, future. Computers and Industrial Engineering 43, 231-249.

Murray, A. T., Grubesic, T. H., Editors, 2007. Critical infrastructure: reliability and vulnerability. Springer, New York.

Murray-Tuite, P. M., Mahmassani, H. S., 2004. Methodology for determining vulnerable links in a transportation network. Transportation Research Record 1882, 88-96.

Nagurney, A., 1999. Network economics: a variational inequality approach, second and revised edition. Kluwer Academic Publishers, Dordrecht, The Netherlands. 
Nagurney, A., 2000. Sustainable transportation networks. Edward Elgar Publishing, Cheltenham, England.

Nagurney, A. 2006a. Supply chain network economics: Dynamics of prices, flows, and profits. Edward Elgar Publishing, Cheltenham, England.

Nagurney, A., 2006b. On the relationship between supply chain and transportation network equilibria: A supernetwork equivalence with computations. Transportation Research E 42, 293-316.

Nagurney, A., 2008. Formulation and analysis of horizontal mergers among oligopolistic firms with insights into the merger paradox: A supply chain network perspective. To appear in Computational Management Science.

Nagurney, A., 2009. A system-optimization perspective for supply chain network integration: The horizontal merger case. Transportation Research E 45, 1-15.

Nagurney, A., 2010. Supply chain network design under profit maximization and oligopolistic competition. Transportation Research E 46, 281-294.

Nagurney, A., Dong, J., 2002. Supernetworks: decision-making for the Information Age. Edward Elgar Publishers, Cheltenham, England.

Nagurney, A., Ke, K., 2003. Financial networks with electronic transactions: modeling, analysis, and computations. Quantitative Finance 3, 71-87.

Nagurney, A., Nagurney, L. S., 2009. Sustainable supply chain network design: A multicriteria perspective. To appear International Journal of Sustainable Engineering.

Nagurney, A., Parkes, D., Daniele, P., 2007. The Internet, evolutionary variational inequality, and the time-dependent Braess Paradox. Computational Management Science 4, 355-375.

Nagurney, A., Qiang, Q., 2007a. A network efficiency measure for congested networks. Europhysics Letters 79, 38005, 1-5.

Nagurney, A., Qiang, Q., 2007b. A transportation network efficiency measure that captures flows, behavior, and costs with applications to network component importance identification and vulnerability. In Proceedings of the 18th Annual POMS Conference, Dallas, Texas.

Nagurney, A., Qiang, Q., 2007c. Robustness of transportation networks subject to degrad- 
able links. Europhysics Letters 80, 68001, 1-6.

Nagurney, A., Qiang, Q., 2008a. A network efficiency measure with application to critical infrastructure networks. Journal of Global Optimization 40, 261-275.

Nagurney, A., Qiang, Q., 2008b. Identification of critical nodes and links in financial networks with intermediation and electronic transactions. In Computational Methods in Financial Engineering. Kontoghiorghes, E. J., Rustem, B., Winker, P., Editors, Springer, Berlin, Germany, pp. 273-297.

Nagurney, A., Qiang, Q., 2008c. An efficiency measure for dynamic networks with application to the Internet and vulnerability analysis. Netnomics 9, 1-20.

Nagurney, A., Qiang, Q., 2009a. Fragile networks: identifying vulnerabilities and synergies in an uncertain world. John Wiley \& Sons, Hoboken, New Jersey.

Nagurney, A., Qiang, Q., 2009b. A relative total cost index for the evaluation of transportation network robustness in the presence of degradable links and alternative travel behavior. International Transactions in Operational Research 16, 49-67.

Nagurney, A., Siokos, S., 1997. Financial networks: statics and dynamics. Springer-Verlag, Heidelberg, Germany.

Nagurney, A., Woolley, T., 2010. Environmental and cost synergy in supply chain network integration in mergers and acquisitions. In Sustainable Energy and Transportation Systems, Proceedings of the 19th International Conference on Multiple Criteria Decision Making, Lecture Notes in Economics and Mathematical Systems. Ehrgott, M., Naujoks, B., Stewart, T. J., Wallenius, J., Editors, Springer, Berlin, Germany, pp. 51-78.

Nagurney, A., Woolley, T., Qiang, Q., 2010. Multiproduct supply chain horizontal network integration: Models, theory, and computational results. International Journal of Operational Research 17, 333-349.

Nagurney, A., Yu, M., Qiang, Q., 2009. Supply chain network design for critical needs with outsourcing. To appear in the Papers in Regional Science.

Nagurney, A., Zhang, D., 1996. Projected dynamical systems and variational inequalities with applications. Kluwer Academic Publishers, Boston, Massachusetts.

Newman, M. E. J., 2004. Analysis of weighted networks. Physical Review E 70, Article No. 
056131.

Newman, M. E. J., Barabási, A. L., Watts, D. J., Editors, 2006. The structure and dynamics of networks. Princeton University Press, Princeton, New Jersey.

Patriksson, M., 1994. The traffic assignment problem - models and methods. VSP, Utrecht, The Netherlands.

Qiang, Q., Nagurney, A., 2008. A unified network performance measure with importance identification and the ranking of network components. Optimization Letters 2, 127-142.

Qiang, Q., Nagurney, A., Dong, J., 2009. Modeling of supply chain risk under disruptions with performance measurement and robustness analysis. In Managing supply chain risk and vulnerability: tools and methods for supply chain decision makers. Wu, T., Blackhurst, J., Editors, Springer, London, England, pp. 91-111.

Ran, B., Boyce, D. E., 1996. Modeling dynamic transportation networks. Springer-Verlag, Berlin, Germany.

Reuters.com, 2010. Global M\&A investment lowest since crisis onset: OECD. March 23.

Roughgarden, T., 2003. The price of anarchy is independent of the network topology. Journal of Computer and System Sciences 67, 341-364.

Roughgarden, T., 2005. Selfish routing and the price of anarchy. MIT Press, Cambridge, Massachusetts.

Sahadi, J., 2009. Stimulus: now for the hard part. CNNMoney.com, February 17.

Schulz, C., 2007. Identification of critical transportation infrastructures. Forum DKKV/CEDM, Disaster Reduction in Climate Change, Karlsruhe University, Germany.

Sheffi, Y., 1985. Urban transportation networks - equilibrium analysis with mathematical programming methods. Prentice-Hall, Englewood Cliffs, New Jersey.

Sheffi, Y., 2005. The resilient enterprise: overcoming vulnerability for competitive advantage. MIT Press, Cambridge, Massachusetts.

Smith, M. J., 1979. Existence, uniqueness, and stability of traffic equilibria. Transportation Research B 13, 259-304. 
Soylu, A., Oruc, C., Turkay, M., Fujita, K., Asakura, T., 2006. Synergy analysis of collaborative supply chain management in energy systems using multi-period MILP. European Journal of Operational Research 174, 387-403.

Taylor, M. A. P., Sekhar, V. C., D'Este, G. M., 2006. Application of accessibility based methods for vulnerability analysis of strategic road networks. Networks and Spatial Economics 6, 267-291.

U.S. Department of Transportation Federal Highway Administration, 2004. Highway statistics 2004. http://www.fhwa.dot.gov Accessed on January 15, 2008.

Van Wassenhove, L. N., 2006. Humanitarian logistics: Supply chain management in high gear. Journal Operational Research Society 57, 475-489.

Walsh, J. R., 2009. Route optimization: how efficient will the proposed north Dublin metro be? ERCIM News 79, October, 45-46.

Wardrop, J. G., 1952. Some theoretical aspects of road traffic research. In Proceedings of the Institution of Civil Engineers, Part II 1, pp. 325-378.

Werdigier, J., Cowell, A., Clark, N., 2010. Airport crisis spreads as ash moves east. The New York Times, April 17.

Wu, K., Nagurney, A., Liu, Z., Stranlund, J. K., 2006. Modeling generator power plant portfolios and pollution taxes in electric power supply chain networks: A transportation network equilibrium transformation. Transportation Research D 11, 171-190. 